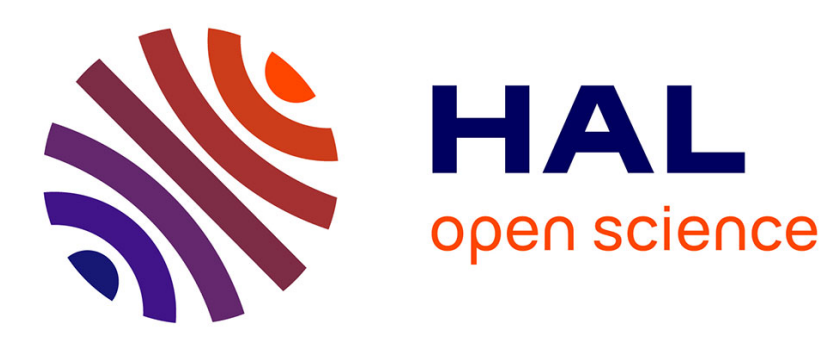

\title{
Reduction of the resonance error. Part 1: Approximation of homogenized coefficients
}

\author{
Antoine Gloria
}

\section{To cite this version:}

Antoine Gloria. Reduction of the resonance error. Part 1: Approximation of homogenized coefficients. Mathematical Models and Methods in Applied Sciences, 2011, 21 (8), pp.1601-1630. 10.1142/S0218202511005507 . inria-00457159

\section{HAL Id: inria-00457159 \\ https://hal.inria.fr/inria-00457159}

Submitted on 16 Feb 2010

HAL is a multi-disciplinary open access archive for the deposit and dissemination of scientific research documents, whether they are published or not. The documents may come from teaching and research institutions in France or abroad, or from public or private research centers.
L'archive ouverte pluridisciplinaire HAL, est destinée au dépôt et à la diffusion de documents scientifiques de niveau recherche, publiés ou non, émanant des établissements d'enseignement et de recherche français ou étrangers, des laboratoires publics ou privés. 


\title{
REDUCTION OF THE RESONANCE ERROR - PART 1: APPROXIMATION OF HOMOGENIZED COEFFICIENTS
}

\author{
ANTOINE GLORIA
}

\begin{abstract}
This paper is concerned with the approximation of effective coefficients in homogenization of linear elliptic equations. One common drawback among numerical homogenization methods is the presence of the so-called resonance error, which roughly speaking is a function of the ratio $\varepsilon / \eta$, where $\eta$ is a typical macroscopic lengthscale and $\varepsilon$ is the typical size of the heterogeneities. In the present work, we propose an alternative for the computation of homogenized coefficients (or more generally a modified cell-problem), which is a first brick in the design of effective numerical homogenization methods. We show that this approach drastically reduces the resonance error in some standard cases.
\end{abstract}

Keywords: numerical homogenization, resonance error, effective coefficients.

2000 Mathematics Subject Classification: 35B27, 60F99.

\section{INTRODUCTION}

This paper deals with numerical homogenization. In essence, numerical homogenization techniques aim at computing solutions to partial differential equations whose coefficients depend on a small parameter denoted by $\varepsilon$, without resolving all the details at the scale $\varepsilon$ (see for instance [15, 16] for the multiscale finite element method, [6, 5] for the heterogeneous multiscale method, and [8] for a general analysis). In the case of periodic coefficients, it amounts to approximating the solution to the homogenized problem.

To be more precise, let us consider the scalar linear elliptic equation

$$
\left\{\begin{aligned}
-\nabla \cdot A_{\varepsilon}(x) \nabla u_{\varepsilon}(x) & =f(x) & & \text { in } D \\
u_{\varepsilon}(x) & =0 & & \text { on } \partial D
\end{aligned}\right.
$$

on a domain $D$ with suitable assumptions on $A_{\varepsilon}$ and $f$. Assume furthermore that $A_{\varepsilon}$ is symmetric and can be homogenized in the following sense: There exists $A_{\text {hom }}$ such that the solution $u_{\varepsilon}$ to (1.1) converges to the solution $u_{\text {hom }}$ to

$$
\left\{\begin{aligned}
-\nabla \cdot A_{\mathrm{hom}}(x) \nabla u_{\mathrm{hom}}(x) & =f(x) & & \text { in } D \\
u_{\mathrm{hom}}(x) & =0 & & \text { on } \partial D
\end{aligned}\right.
$$

for all suitable $f$ (as well as the flux $A_{\varepsilon} \nabla u_{\varepsilon}$ to $A_{\text {hom }} \nabla u_{\text {hom }}$ ). Such a homogenization property typically arises when $A_{\varepsilon}$ is the combination of a smooth function and an oscillating part at scale $\varepsilon>0$. Unfortunately, $A_{\text {hom }}$ is not explicit in general. The aim of numerical homogenization can now be rephrased as: Given $A_{\varepsilon}$ (and not $A_{\text {hom }}$ ), approximate $u_{\text {hom }}$ without solving (1.1) at scale $\varepsilon$ ? 
A very general approach is as follows. For all $\eta \geq \varepsilon>0$, let $A_{\eta, \varepsilon}$ be defined by

$$
\xi \cdot A_{\eta, \varepsilon}(x) \xi:=\inf \left\{f_{D \cap B(x, \eta)}(\xi+\nabla \phi) \cdot A_{\varepsilon}(y)(\xi+\nabla \phi) d y, \phi \in H_{0}^{1}(D \cap B(x, \eta))\right\}
$$

for all $\xi$, where $B(x, \eta)$ is the ball centered at $x$ and of radius $\eta$. An approximation of $u_{\text {hom }}$ is then given by $u_{\eta, \varepsilon}$, solution to

$$
\left\{\begin{aligned}
-\nabla \cdot A_{\eta, \varepsilon}(x) \nabla u_{\eta, \varepsilon}(x) & =f(x) & & \text { in } D \\
u_{\eta, \varepsilon}(x) & =0 & & \text { on } \partial D .
\end{aligned}\right.
$$

In particular (see [8] for general results), we have

$$
\lim _{\eta \rightarrow 0} \lim _{\varepsilon \rightarrow 0} A_{\eta, \varepsilon}=A_{\text {hom }}
$$

in $L^{p}(D)$ for all $p<\infty$, and

$$
\lim _{\eta \rightarrow 0} \lim _{\varepsilon \rightarrow 0}\left\|u_{\eta, \varepsilon}-u_{\text {hom }}\right\|_{H^{1}(D)}=0 .
$$

The main difference between (1.4) and (1.1) is that $A_{\eta, \varepsilon}$ is expected not to oscillate at scale $\varepsilon$, which is a big advantage for the numerical practice.

In order to make a numerical analysis of the above method, and in particular to quantify the convergences $(1.5) \&(1.6)$, we turn to the simplest case possible: $A_{\varepsilon}(x)=A(x / \varepsilon)$, where $A$ is periodic. Then, one can prove that

$$
A_{\eta, \varepsilon}=A_{\mathrm{hom}}+O(\varepsilon / \eta)
$$

and

$$
\left\|u_{\eta, \varepsilon}-u_{\mathrm{hom}}\right\|_{H^{1}(D)}=O(\varepsilon / \eta) .
$$

The term $O(\varepsilon / \eta)$ is called the resonance error. In practice, that is when (1.3) and (1.4) are solved numerically (finite element, finite volume, finite difference, FFT methods etc.), the dominant term in the overall error can precisely be the resonance error $O(\varepsilon / \eta)$. Several refinements have then been introduced to reduce this error (such as oversampling in [17], and filtering in [22]). However, as shown in [9], even with these refinements, although the prefactor may have been reduced, the error is still of order $O(\varepsilon / \eta)$.

The aim of this paper is to reduce the order of magnitude of the resonance error. Let us first perform a change of variables to make the lengthscale of the oscillations of $A_{\varepsilon}$ be of order 1 , and rewrite (1.3) as

$$
\begin{array}{r}
\xi \cdot A_{\eta, \varepsilon}(x) \xi:=\inf \left\{\int_{\frac{D-x}{\varepsilon} \cap B\left(0, \frac{\eta}{\varepsilon}\right)}\left(\xi+\nabla \phi_{\eta, \varepsilon}\right) \cdot A(y)\left(\xi+\nabla \phi_{\eta, \varepsilon}\right) d y,\right. \\
\left.\phi_{\eta, \varepsilon} \in H_{0}^{1}\left(\frac{D-x}{\varepsilon} \cap B\left(0, \frac{\eta}{\varepsilon}\right)\right)\right\},
\end{array}
$$

where $A(y):=A_{\varepsilon}(x+\varepsilon y)$. Essentially, (1.9) can be seen as an approximation of the averaged energy density

$$
\mathcal{M}((\xi+\nabla \phi) \cdot A(y)(\xi+\nabla \phi)):=\lim _{R \rightarrow \infty} \frac{1}{\left|Q_{R}\right|} \int_{Q_{R}}(\xi+\nabla \phi) \cdot A(y)(\xi+\nabla \phi) d x
$$

where $Q_{R}=(-R, R)^{d}$, and $\phi$ is a solution (in a suitable sense) to

$$
-\nabla \cdot A(\xi+\nabla \phi)_{2}=0 \quad \text { in } \mathbb{R}^{d} .
$$


This article is devoted to the approximation of (1.10). Our method is inspired by the analysis by Otto and the author in [10, 11, 12] and a recent article [3] by Blanc \& Le Bris. We show that for some benchmark tests the proposed method effectively reduces the resonance error. In particular, in the periodic case, estimate (1.7) is replaced by

$$
A_{\eta, \varepsilon}=A_{\mathrm{hom}}+O(\varepsilon / \eta)^{p}
$$

for all $p<4$. In the case of stochastic homogenization of discrete elliptic equations, the method performs also quite well, as recently proved by Otto and the author in [10, 11, 12]. The coupling with numerical homogenization methods will be the object of a subsequent work.

The article is organized as follows. In Section 2, we precisely define the resonance error when approximating homogenized coefficients and introduce our new method. The convergence of the method is analyzed in Section 3 for periodic coefficients (and results on the stochastic case are recalled). A numerical study completes the analysis in Section 4.

We make use of the following notation:

- $d \geq 1$ is the dimension;

- $\lesssim$ and $\gtrsim$ stand for $\leq$ and $\geq$ up to a multiplicative constant which only depends on the dimension $d$ and the constants $\alpha, \beta$ (see Definition 2 below) if not otherwise stated;

- when both $\lesssim$ and $\gtrsim$ hold, we simply write $\sim$;

- we use $\gg$ instead of $\gtrsim$ when the multiplicative constant is (much) larger than 1.

\section{Resonance ERRor AND PROPOSED STRATEGy}

In this section, we assume (as this is the case in periodic, quasi-periodic or stochastic homogenization) that the corrector problem is posed on $\mathbb{R}^{d}$, the corrector $\phi$ being solution to

$$
-\nabla \cdot A(\xi+\nabla \phi)=0 \quad \text { in } \mathbb{R}^{d}
$$

where $A$ is a symmetric matrix. The homogenized coefficients are then given by

$$
\xi \cdot A_{\mathrm{hom}} \xi=\mathcal{M}\{(\xi+\nabla \phi) \cdot A(\xi+\nabla \phi)\},
$$

where $\mathcal{M}\{\cdot\}$ is the average operator on $\mathbb{R}^{d}$ :

$$
\mathcal{M}\{\mathcal{E}\}:=\lim _{R \rightarrow \infty} \frac{1}{\left|Q_{R}\right|} \int_{Q_{R}} \mathcal{E}(x) d x,
$$

and $Q_{R}:=(-R, R)^{d}$. Such quantities are well-defined in periodic, quasi-periodic and stochastic homogenization (see for instance the monograph [18]).

To numerically compute $A_{\text {hom }}$ via (2.2), one needs to approximate the corrector field $\phi$ and the average operator $\mathcal{M}\{\cdot\}$. These two approximations lead to the so-called resonance error.

2.1. The resonance error. The simplest way to approximate $\phi$ and $\mathcal{M}\{\cdot\}$ consists in solving (2.1) on a large domain $Q_{R}=(-R, R)^{d}$ (with suitable boundary conditions, say homogeneous Dirichlet), and taking the average of the energy density on $Q_{R}$. Doing so, and recalling [9, Section 3.1], we make at least two errors: 
- A geometric error $\left(Q_{R}\right.$ is not necessarily a multiple of the unit cell in the periodic case, so that even if the solution on $Q_{R}$ were the true corrector, the average of the energy density on $Q_{R}$ would not coincide with its average on a periodic cell);

- An error related to the boundary condition (we do not know a priori what to impose on $\partial Q_{R}$, and consequently we make an error on the corrector).

More precisely, $A_{\text {hom }}$ is approximated by

$$
\xi \cdot A_{R} \xi:=\frac{1}{\left|Q_{R}\right|} \int_{Q_{R}}\left(\xi+\nabla \phi_{R}(x)\right) \cdot A(x)\left(\xi+\nabla \phi_{R}(x)\right) d x,
$$

where $\phi_{R}$ is the unique solution in $H_{0}^{1}\left(Q_{R}\right)$ to

$$
-\nabla \cdot A\left(\xi+\nabla \phi_{R}\right)=0 \text {. }
$$

In the periodic case, the associated error is of the order

$$
\left|A_{R}-A_{\text {hom }}\right| \sim \frac{1}{R}
$$

in any dimension. Using oversampling and filtering methods, that is setting

$$
\xi \cdot \tilde{A}_{R} \xi:=\int_{Q_{R}}\left(\xi+\nabla \phi_{R}(x)\right) \cdot A(x)\left(\xi+\nabla \phi_{R}(x)\right) \mu_{R}(x) d x,
$$

where $\mu_{R}$ is typically a smooth non-negative mask such that

$$
\begin{aligned}
\left\|\nabla \mu_{R}\right\|_{L^{\infty}} & \lesssim R^{-d-1} \\
\int_{Q_{R}} \mu_{R}(x) d x & =1 \\
\mu_{R \mid Q_{R} \backslash Q_{R / 2}} & \equiv 0
\end{aligned}
$$

we may hope to reduce both sources of the error. However, the overall error is still of order

$$
\left|\tilde{A}_{R}-A_{\mathrm{hom}}\right| \sim \frac{1}{R}
$$

in any dimension, as already noticed by E \& Yue in [22]. Only the prefactor may have been reduced (see a related numerical test on Figure 7 ).

In the following subsection, we propose to treat separately the two sources of error. The geometric error is an error localized at the boundary, and a filtering method with a suitable mask is enough to significantly reduce it. The error we make on the boundary conditions has however non-local effects due to the poor decay of the Green function of the Laplace operator. To reduce this effect, it is natural to add a zero-order term to the equation, which makes the associated Green function decay exponentially fast. This allows to drastically reduce the spurious effect of the boundary condition away from a boundary layer. Yet, this modifies the corrector equation and introduces a bias, which has to be quantified. The last task consists in suitably choosing the different parameters at stake.

2.2. Proposed strategy. As a proxy for the corrector field $\phi$ solution to (2.1), we consider $\phi_{T, R}$, solution to

$$
\left\{\begin{array}{rll}
T^{-1} \phi_{T, R}-\nabla \cdot A\left(\xi+\nabla \phi_{T, R}\right) & =0 & \text { in } Q_{R}, \\
\phi_{T, R} & =0 & \text { on } \partial Q_{R},
\end{array}\right.
$$


where $T>0$ controls the importance of the zero-order term and $R>0$ is the size of the finite domain $Q_{R}$. We then approximate the homogenized coefficient by taking the filtered average

$$
\xi \cdot A_{T, R, L} \xi:=\int_{Q_{R}}\left(\xi+\nabla \phi_{T, R}(x)\right) \cdot A(x)\left(\xi+\nabla \phi_{T, R}(x)\right) \mu_{L}(x) d x,
$$

where $\mu_{L}$ is a smooth mask (whose properties will be fixed in Definition 1) supported in $Q_{L}=(-L, L)^{d}, L \leq R$. Note that we do not consider the full energy associated with (2.4) and disregard the contribution of the zero-order term $T^{-1} \phi_{T, R}(x)^{2}$ in (2.5). The reason for this choice is made clear in the following discussion.

In order to choose the parameters $T, R, L$ properly, we first make a coarse analysis of the error, that we split into three parts:

$$
\left|A_{T, R, L}-A_{\mathrm{hom}}\right| \leq\left|A_{T}-A_{\mathrm{hom}}\right|+\left|A_{T, L}-A_{T}\right|+\left|A_{T, R, L}-A_{T, L}\right|,
$$

where the different quantities are described below. We first define an approximate corrector $\phi_{T}$, solution to

$$
T^{-1} \phi_{T}-\nabla \cdot A\left(\xi+\nabla \phi_{T}\right)=0 \quad \text { in } \mathbb{R}^{d} .
$$

Such a function is well-defined in the periodic, quasi-periodic and stochastic cases. The matrix $A_{T}$ is then characterized by

$$
\xi \cdot A_{T} \xi:=\mathcal{M}\left\{\left(\xi+\nabla \phi_{T}\right) \cdot A\left(\xi+\nabla \phi_{T}\right)\right\} .
$$

The error term $\left|A_{T}-A_{\text {hom }}\right|$ depends on $T$ and on the structure properties of $A$ (periodicity, quasi-periodicity, stochastic stationarity...). As will be seen in the proof for the periodic case, we have the following universal lower bound:

$$
\left|A_{T}-A_{\mathrm{hom}}\right| \gtrsim T^{-2} .
$$

Note that if we had added the zero order term $T^{-1} \mathcal{M}\left\{\phi_{T}^{2}\right\}$ in the definition of $A_{T}$, the error $\left|A_{T}-A_{\text {hom }}\right|$ would have been at least of order $T^{-1}$. This motivates the definition of $A_{T}$, and more generally of (2.5). The second error term $\left|A_{T}-A_{T, L}\right|$, where

$$
\xi \cdot A_{T, L} \xi:=\int_{Q_{L}}\left(\xi+\nabla \phi_{T}(x)\right) \cdot A(x)\left(\xi+\nabla \phi_{T}(x)\right) \mu_{L}(x) d x,
$$

is partly geometric. It can be reduced if a suitable mask $\mu_{L}$ is used. Note that, as opposed to $\phi_{T, R}, \phi_{T}$ has the same structure property as $A$ (periodicity, quasi-periodicity, stationarity...), which is a big advantage for the analysis (it is crucial for the analysis of the discrete stochastic case in [10]). The last error term $\left|A_{T, R, L}-A_{T, L}\right|$ can be treated using standard elliptic estimates. It is essentially of infinite order in units of $(R-L) / \sqrt{T}$.

The combination of these three error terms allows us to make reasonable choices for $L, R, T: R \sim L$ and $L \gg \sqrt{T}$. The error is at least of order $T^{-2}$, hence $R^{-4}$ (recall that $R$ quantifies the cost to compute $\left.\phi_{T, R}\right)$. As will be seen in the following section, this strategy outperforms (both in terms of precision and/or computational cost) most of the other existing methods. 


\section{AnAlysis OF SOME STANDARD CASES}

Before turning to the analysis proper, let us make precise the form of the masks we use.

Definition 1. A function $\mu:[-1,1] \rightarrow \mathbb{R}^{+}$is said to be a filter of order $p \geq 0$ if

(i) $\mu \in C^{p}([-1,1]) \cap W^{p+1, \infty}((-1,1))$,

(ii) $\int_{-1}^{1} \mu(x) d x=1$,

(iii) $\mu^{(k)}(-1)=\mu^{(k)}(1)=0$ for all $k \in\{0, \ldots, p-1\}$.

The associated mask $\mu_{L}:[-L, L]^{d} \rightarrow \mathbb{R}^{+}$in dimension $d \geq 1$ is then defined for all $L>0$ by

$$
\mu_{L}(x):=L^{-d} \prod_{i=1}^{d} \mu\left(L^{-1} x_{i}\right),
$$

where $x=\left(x_{1}, \ldots, x_{d}\right) \in \mathbb{R}^{d}$.

3.1. The periodic case. Let us first introduce the class of matrices we consider.

Definition 2. For all $d \geq 1, \beta \geq \alpha>0$, we define $\mathcal{A}_{\alpha \beta}$ as the set of those symmetric matrices of order $d$ such that for all $x \in \mathbb{R}^{d}$,

$$
\begin{aligned}
x \cdot A x & \geq \alpha|x|^{2}, \\
|A x| & \leq \beta|x| .
\end{aligned}
$$

For all $D$ open subset of $\mathbb{R}^{d}$, and all $A: D \rightarrow \mathcal{A}_{\alpha \beta}$, we use the shorthand notation $A \in \mathcal{A}_{\alpha \beta}$.

Theorem 1. Let $d \geq 2, A \in \mathcal{A}_{\alpha \beta}$ be $Q$-periodic, $\mu$ be a filter of order $p \geq 0$, and $A_{\mathrm{hom}}$ and $A_{T, R, L}$ be the homogenized matrix and its approximation (2.5) respectively, where $R^{2} \gtrsim T \gtrsim R, R \geq L \sim R \sim R-L$. Then, there exists $c>0$ depending only on $\alpha, \beta$ and $d$ such that we have

$$
\left|A_{T, R, L}-A_{\mathrm{hom}}\right| \lesssim L^{-(p+1)}+T^{-2}+T^{1 / 4} \exp \left(-c \frac{R-L}{\sqrt{T}}\right) .
$$

We postpone the proof to Subsection 3.3, and directly turn to an application of Theorem 1. For $p \geq 3$, the rate in (3.1) is controlled by the last two terms. In particular the last term requires $T$ to be such that $L \gg \sqrt{T}$. A possible choice is then given by

- $T=L^{2}(\ln L)^{-4}$,

- $R=3 L / 2$,

for which (3.1) reads:

$$
\left|A_{T, R, L}-A_{\text {hom }}\right| \lesssim R^{-4} \ln ^{8} R
$$

Whereas estimate (2.3) in the basic approach of Subsection 2.1 is of order 1, the present approach yields an estimate (3.2) up to order $4^{-}$.

Note that recently, Blanc \& Le Bris have developed another strategy in [3], where essentially the mask is introduced in the very definition of the bilinear form associated with the equation, and not as a post-processing tool. Their formal analysis and numerical tests show a convergence of order 2 in the periodic case, which cannot be improved in general. The better result (3.2) of the present strategy is mainly due to the efficient treatment, by the zero-order term, of the spurious effects of the boundary conditions. 
3.2. A stochastic example. In this subsection, we quickly review the results obtained by Otto and the author in $[10,11,12]$, since they complement the analysis of the periodic case quite well. In these articles, the elliptic equation is discrete, and $A$ is a set of conductivities on the edges of $\mathbb{Z}^{d}$. The strategy remains the same, and we keep the notation of Subsection 2.1. The results are as follows: Let $d \geq 2, A \in \mathcal{A}_{\alpha \beta}$ be an independently and identically distributed conductivity function in the sense of [10], and $A_{\text {hom }}$, $A_{T}, A_{T, L}$ and $A_{T, R, L}$ be the homogenized matrix and its approximations, where $T>0$, $R \geq L \sim R \sim R-L$ and $\mu_{L}$ as in Definition 1 with $p \geq 1^{1}$. Then, there exist $c>0$ and $q>0$ depending only on $\alpha, \beta$ and $d$ such that we have

$$
\begin{aligned}
& \left|A_{T}-A_{\text {hom }}\right| \quad \lesssim \quad\left\{\begin{array}{lll}
d=2 & : & T^{-1} \ln ^{q} T \\
d=3 & : & T \\
d=4 & : & T \\
d>4 & : & T
\end{array}\right. \\
& \left\langle\left|A_{T, L}-A_{T}\right|^{2}\right\rangle^{1 / 2} \lesssim\left\{\begin{array}{lll}
d=2 & : & L^{-1} \ln ^{q} T \\
d>2 & : & L^{-d / 2}
\end{array}\right. \\
& \left|A_{T, R, L}-A_{T, L}\right| \stackrel{\text { almost surely }}{\lesssim} T^{3 / 4} \exp \left(-c \frac{R-L}{\sqrt{T}}\right),
\end{aligned}
$$

where $\langle\cdot\rangle$ denotes the ensemble average, or equivalently expectation in the underlying probability space. Let us comment on the above results. In the periodic case, the estimate $L^{-(p+1)}$ for the error term $\left|A_{T, L}-A_{T}\right|$ can be made of any order provided the use of an appropriate mask $\mu_{L}$. In the stochastic case, the order of accuracy is naturally limited by the central limit theorem scaling, which we recognise in (3.4) (up to the logarithmic correction for $d=2$ ). As for the periodic case, the estimate (3.5) for the error term $\left|A_{T, R, L}-A_{T, L}\right|$ is of infinite order in units of $(R-L) / \sqrt{T}$ (up to the multiplicative constant $\left.T^{3 / 4}\right)$. Finally, the estimate (3.3) of the error term $\left\langle\left|A_{T}-A_{\text {hom }}\right|^{2}\right\rangle^{1 / 2}$ also saturates at $T^{-2}$ in high dimension, but further depends on the dimension for $d<4$, which is a consequence of the stochastic structure.

In this stochastic example, a natural choice for $T, L, R$ is as follows:

- $T=R$,

- $L=R\left(1-\ln ^{2} R / \sqrt{R}\right)$,

and the global error estimate reads

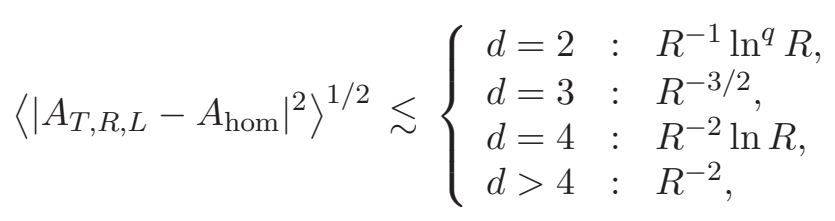

which is sharp.

The extension of these results to the continuous setting is currently under investigation.

\footnotetext{
${ }^{1}$ The role of the mask is different in this case since the conductivities are i. i. d., so that there is a priori no "geometric error" involved. In [10], the use of the mask appears as a technical requirement somewhat unrelated to the previous discussion. The situation would be different in the continuous stochastic case.
} 
3.3. Proof in the periodic case. We divide the proof of Theorem 1 in three steps. Let $H_{\text {per }}^{1}(Q)$ denote the subspace of periodic functions of $H^{1}(Q)$ with zero average, and $\xi \in \mathbb{R}^{d}$ with $|\xi|=1$.

Step 1. Proof of

$$
\left|A_{T}-A_{\mathrm{hom}}\right| \lesssim T^{-2}
$$

We recall that $A_{\text {hom }}$ is given by

$$
\xi \cdot A_{\mathrm{hom}} \xi=f_{Q}(\xi+\nabla \phi) \cdot A(\xi+\nabla \phi)
$$

where $\phi$ is the unique weak solution in $H_{\text {per }}^{1}(Q)$ to

$$
-\nabla \cdot A(\xi+\nabla \phi)=0 .
$$

We have, using equation (3.8) and the symmetry of $A$,

$$
\begin{aligned}
\xi \cdot\left(A_{T}-A_{\mathrm{hom}}\right) \xi & =\int_{Q}\left(\xi+\nabla \phi_{T}\right) \cdot A\left(\xi+\nabla \phi_{T}\right)-(\xi+\nabla \phi) \cdot A(\xi+\nabla \phi) \\
& =\int_{Q}\left(\xi+\nabla \phi_{T}\right) \cdot A \nabla\left(\phi_{T}-\phi\right)+\nabla\left(\phi_{T}-\phi\right) \cdot A(\xi+\nabla \phi) \\
\stackrel{(3.8)}{=} & \int_{Q}\left(\xi+\nabla \phi_{T}\right) \cdot A \nabla\left(\phi_{T}-\phi\right)-\nabla\left(\phi_{T}-\phi\right) \cdot A(\xi+\nabla \phi) \\
& =\int_{Q} \nabla\left(\phi_{T}-\phi\right) \cdot A \nabla\left(\phi_{T}-\phi\right) .
\end{aligned}
$$

Introducing $\psi_{T}$ defined by

$$
\psi_{T}=-T\left(\phi_{T}-\phi\right),
$$

this identity turns into

$$
\xi \cdot\left(A_{T}-A_{\mathrm{hom}}\right) \xi=T^{-2} \int_{Q} \nabla \psi_{T} \cdot A \nabla \psi_{T} .
$$

Note that $\psi_{T}$ is the unique solution in $H_{\mathrm{per}}^{1}(Q)$ to

$$
T^{-1} \psi_{T}-\nabla \cdot A \nabla \psi_{T}=\phi
$$

We then appeal to equation (3.10) in the form of the a priori estimate

$$
T^{-1} \int_{Q} \psi_{T}^{2}+\int_{Q} \nabla \psi_{T} \cdot A \nabla \psi_{T}=\int_{Q} \phi \psi_{T},
$$

which turns into

$$
\int_{Q}\left|\nabla \psi_{T}\right|^{2} \lesssim \int_{Q} \phi^{2}
$$

by uniform ellipticity of $A$ and Poincaré's inequality in $H_{\text {per }}^{1}(Q)$. Combined with (3.9), this yields

$$
\begin{aligned}
\left|\xi \cdot\left(A_{T}-A_{\mathrm{hom}}\right)\right| \xi & \lesssim T^{-2} \int_{Q} \phi^{2} \\
& \lesssim T^{-2} \int_{Q}|\nabla \phi|^{2} \\
& \lesssim T^{-2}|\xi|^{2}=T^{-2}
\end{aligned}
$$


using Poincaré's inequality in $H_{\text {per }}^{1}(Q)$, and an a priori estimate on $\nabla \phi$. This proves (3.7). Step 2. Proof of

$$
\left|A_{T}-A_{T, L}\right| \lesssim L^{-(p+1)} .
$$

Estimate (3.11) would be a consequence of the following lemma if the energy density

$$
\mathcal{E}_{T}: x \mapsto\left(\xi+\nabla \phi_{T}(x)\right) \cdot A(x)\left(\xi+\nabla \phi_{T}(x)\right),
$$

which is a $Q$-periodic function, were square integrable uniformly in $T$.

Lemma 1. Let $\mu$ be a filter of order $p \geq 0$ according to Definition 1. Then, for all $\phi \in L_{\mathrm{per}}^{2}(Q)$ and all $L>0$, we have

$$
\left|\int_{Q_{L}} \phi(x) \mu_{L}(x) d x-\mathcal{M}(\phi)\right| \lesssim L^{-(p+1)}\|\phi\|_{L^{2}(Q)},
$$

where $Q_{L}=(-L, L)^{d}$ and $\mu_{L}(x):=L^{-d} \prod_{i=1}^{d} \mu\left(L^{-1} x_{i}\right), x=\left(x_{1}, \ldots, x_{d}\right)$. The constant in (3.12) only depends on $p$ and $\mu$.

Note that Lemma 1 holds for general periodic functions (not necessarily $Q$-periodic).

Yet, $\mathcal{E}_{T}$ is only in $L^{1}(Q)$. As proved in Appendix A, the higher integrability $\mathcal{E}_{T} \in L^{q}(Q)$ for some $q>1$ is enough to conclude. This higher integrability of the energy density is a standard consequence of Meyers' estimate (see the original article by Meyers [19], and its use for homogenization problems by Murat and Tartar in [20]), noting that $q$ depends on $\alpha, \beta$, and $d$, but not on $T$ (see for instance the argument in [10, Proof of Lemma 9] in the discrete case).

Step 3. Proof of

$$
\left|A_{T, R, L}-A_{T, L}\right| \lesssim T^{1 / 4} \exp \left(-c \frac{R-L}{\sqrt{T}}\right) .
$$

Our proof of (3.13), which is self-contained, sligthly departs from the approach by Bourgeat $\&$ Piatnitski in [4]. The argument is based on the exponential decay of the Green function, which is the object of the following auxiliary lemma.

Lemma 2. Let $G_{T}$ be the Green function associated with the operator $\left(T^{-1}-\nabla \cdot A \nabla\right)$ on $\mathbb{R}^{d}$. Then there exists $c>0$ depending only on $\alpha, \beta$ and $d$ such that the following pointwise estimate holds for all $|x-y| \gtrsim \sqrt{T}$ :

$$
G_{T}(x, y) \lesssim \frac{1}{|x-y|^{d-2}} \exp \left(-c \frac{|x-y|}{\sqrt{T}}\right) .
$$

Although this result is common knowledge, we did not find any precise reference for it. A proof is given in Appendix B.

By definition of $\phi_{T}$ and $\phi_{T, R}$, we have

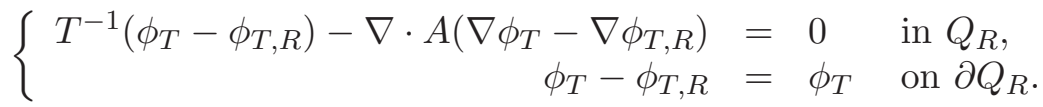

We then consider a lifting $\phi_{1}$ of $\phi_{T \mid \partial Q_{R}}$ on $Q_{R}$ such that $\phi_{1 \mid Q_{R-1}} \equiv 0$, and $\left\|\phi_{1}\right\|_{H^{1}\left(Q_{R}\right)}^{2} \lesssim$ $R^{d-1}\left\|\phi_{T}\right\|_{H^{1}(Q)}^{2}$ (recall that $\phi_{T}$ is $Q$-periodic). The function $\phi_{2}:=\phi_{T}-\phi_{T, R}-\phi_{1}$ then satisfies the equation

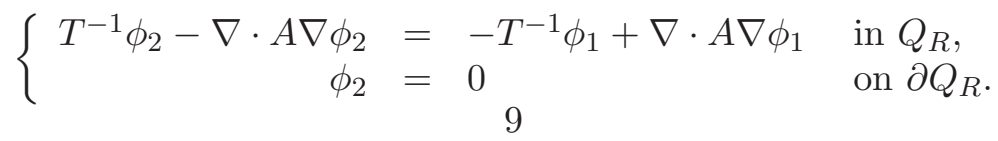


Let $G_{T, R}: Q_{R} \times Q_{R} \rightarrow \mathbb{R}^{+}$be the Green function associated with the operator $\left(T^{-1}\right.$ $\nabla \cdot A \nabla)$ on $Q_{R}$ with homogeneous Dirichlet boundary conditions. The function $\phi_{2}$ can be written as

$$
\phi_{2}(x)=-\int_{Q_{R}}\left(T^{-1} \phi_{1}(y) G_{T, R}(x, y)+\nabla G_{T, R}(x, y) \cdot A(y) \nabla \phi_{1}(y)\right) d y .
$$

By Cauchy-Schwarz' inequality, this turns into

$$
\begin{array}{r}
\mid \phi_{2}(x) \leq\left\|\phi_{1}\right\|_{H^{1}\left(Q_{R}\right)}\left(T^{-1}\left(\int_{Q_{R} \backslash Q_{R-1}} G_{T, R}(x, y)^{2} d y\right)^{1 / 2}+\right. \\
\left.\left(\int_{Q_{R} \backslash Q_{R-1}}\left|\nabla G_{T, R}(x, y)\right|^{2} d y\right)^{1 / 2}\right) .
\end{array}
$$

To control the first term of the r. h. s. of (3.16), we use Lemma 2, which, combined with the maximum principle, yields an estimate for $0 \leq G_{T, R} \leq G_{T}$. For the second term of the r. h. s. of (3.16), we use Cacciopoli's inequality. To this aim, let $\eta: Q_{R} \rightarrow \mathbb{R}^{+}$be a function of class $C^{1}$. We multiply the defining equation for $G_{T, R}$ by $\eta^{2} G_{T, R}$, integrate on $Q_{R}$, and obtain after integration by parts

$$
\begin{gathered}
0=T^{-1} \int_{Q_{R}} \eta^{2}(y) G_{T, R}(x, y)^{2} d y+\int_{Q_{R}} \nabla G_{T, R}(x, y) \cdot A(y) \nabla\left(\eta(y)^{2} G_{T, R}(x, y)\right) d y \\
=T^{-1} \int_{Q_{R}} \eta^{2}(y) G_{T, R}(x, y)^{2} d y+\int_{Q_{R}} \nabla\left(\eta(y) G_{T, R}(x, y)\right) \cdot A(y) \nabla\left(\eta(y) G_{T, R}(x, y)\right) d y \\
\quad-\int_{Q_{R}} G_{T, R}(x, y)^{2} \nabla \eta(y) \cdot A(y) \nabla \eta(y) d y
\end{gathered}
$$

provided $\eta_{\mid \mathcal{N}(x)} \equiv 0$ on an open neighborhood $\mathcal{N}(x)$ of $x$. Hence, by the uniform bounds on $A$,

$$
\int_{Q_{R}}\left|\nabla\left(\eta(y) G_{T, R}(x, y)\right)\right|^{2} d y \lesssim \int_{Q_{R}} G_{T, R}(x, y)^{2}|\nabla \eta(y)|^{2} d y
$$

Taking now $\sqrt{T} \leq \rho \leq R / 2$, and $\eta: Q_{R} \rightarrow[0,1]$ such that

$$
\begin{aligned}
& \text { for } y \in Q_{R-\rho / 2} \quad: \quad \eta(y)=0, \\
& \text { for } y \in Q_{R} \backslash Q_{R-1}: \quad \eta(y)=1 \text {, } \\
& \text { for } y \in Q_{R} \quad: \quad|\nabla \eta(y)| \lesssim \rho^{-1} \text {, }
\end{aligned}
$$

(3.17) turns into

$$
\left.\int_{Q_{R} \backslash Q_{R-1}} \mid \nabla G_{T, R}(x, y)\right)\left.\right|^{2} d y \lesssim \rho^{-2} \int_{Q_{R} \backslash Q_{R-\rho / 2}} G_{T, R}(x, y)^{2} d y
$$


for all $x \in Q_{R-\rho}$. Inserting now (3.18) into (3.16) and using Lemma 2 to control $G_{T, R}$, we obtain for all $x \in Q_{R-\rho}$

$$
\begin{aligned}
&\left|\phi_{2}(x)\right| \lesssim\left\|\phi_{1}\right\|_{H^{1}\left(Q_{R}\right)}\left(T^{-1}\left(\int_{Q_{R} \backslash Q_{R-1}} G_{T, R}(x, y)^{2} d y\right)^{1 / 2}+\right. \\
&\left.\rho^{-1}\left(\int_{Q_{R} \backslash Q_{R-\rho / 2}} G_{T, R}(x, y)^{2} d y\right)^{1 / 2}\right) \\
& \lesssim R^{(d-1) / 2}\left(T^{-1} R^{(d-1) / 2} \rho^{2-d} \exp \left(-c \frac{\rho}{\sqrt{T}}\right)+\right. \\
& \leq\left(\frac{R^{d-1}}{\rho^{d-2} T}+\frac{R^{d-1 / 2}}{\rho^{d-1}}\right) \exp \left(-c \frac{\rho}{2 \sqrt{T}}\right)
\end{aligned}
$$

Combined with the assumption $R^{2} \gtrsim T \gtrsim R$ and taking $\rho=R / 4$, this inequality can be further simplified to

$$
\left|\phi_{2}(x)\right| \lesssim T^{1 / 4} \exp \left(-c \frac{\rho}{2 \sqrt{T}}\right),
$$

for some slightly smaller $c>0$, noting that

$$
\rho^{1 / 2} \exp \left(-c \frac{\rho}{2 \sqrt{T}}\right)=T^{1 / 4}\left(\frac{\rho}{\sqrt{T}}\right)^{1 / 2} \exp \left(-c \frac{\rho}{2 \sqrt{T}}\right) .
$$

Hence,

$$
\int_{Q_{R-\rho}} \phi_{2}(x)^{2} d x \lesssim(R-\rho)^{d} \sqrt{T} \exp \left(-c \frac{\rho}{\sqrt{T}}\right) .
$$

Another use of Caccciopoli's inequality, this time for $\phi_{2}$ (recall that the r. h. s. of (3.15) vanishes identically in $Q_{R-1}$ ), then yields

$$
\int_{Q_{R-\rho}}\left|\nabla \phi_{2}(x)\right|^{2} d x \lesssim(R-\rho)^{d} \sqrt{T} \exp \left(-c \frac{\rho}{\sqrt{T}}\right)
$$

as well. 
We are now in position to conclude the proof of (3.13):

$$
\begin{aligned}
\mid \xi & \cdot\left(A_{T, L, R}-A_{T, L}\right) \xi \mid \\
& =\left|f_{Q_{L}}\left(\left(\xi+\nabla \phi_{T, R}\right) \cdot A\left(\xi+\nabla \phi_{T, R}\right)-\left(\xi+\nabla \phi_{T}\right) \cdot A\left(\xi+\nabla \phi_{T}\right)\right) \mu_{L}(x) d x\right| \\
& =\left|f_{Q_{L}}\left(\left(\nabla \phi_{T, R}-\nabla \phi_{T}\right) \cdot A\left(\xi+\nabla \phi_{T, R}\right)-\left(\xi+\nabla \phi_{T}\right) \cdot A\left(\nabla \phi_{T}-\nabla \phi_{T, R}\right)\right) \mu_{L}(x) d x\right| \\
& \lesssim\left(f_{Q_{L}}\left|\nabla \phi_{T, R}-\nabla \phi_{T}\right|^{2} d x\right)^{1 / 2}\left(\left(f_{Q_{L}}\left|\xi+\nabla \phi_{T}\right|^{2} d x\right)^{1 / 2}+\left(f_{Q_{L}}\left|\xi+\nabla \phi_{T, R}\right|^{2} d x\right)^{1 / 2}\right) \\
& =\left(f_{Q_{L}}\left|\nabla \phi_{2}\right|^{2} d x\right)^{1 / 2}\left(\left(f_{Q_{L}}\left|\xi+\nabla \phi_{T}\right|^{2} d x\right)^{1 / 2}+\left(f_{Q_{L}}\left|\xi+\nabla \phi_{T, R}\right|^{2} d x\right)^{1 / 2}\right) .
\end{aligned}
$$

Combined with the a priori estimates

$$
\begin{aligned}
\left\|\nabla \phi_{T}\right\|_{H^{1}(Q)} & \lesssim 1, \\
\left\|\nabla \phi_{T, R}\right\|_{H^{1}\left(Q_{L}\right)}^{2} \leq\left\|\nabla \phi_{T, R}\right\|_{H^{1}\left(Q_{R}\right)}^{2} & \lesssim R^{d},
\end{aligned}
$$

and (3.20) with $R-\rho=L$, this proves the claim of Step 3, and concludes the proof of Theorem 1.

\section{NUMERICAL STUDY}

In this section, we present numerical tests which show that Theorem 1 is sharp. Note however that Theorem 1 only gives an asymptotic rate of convergence, whereas in practice one is interested in values of the order $1 \leq R \leq 50$. In this case, it is not clear how the method behaves. We therefore present numerical tests in the two different regimes: $R \gg 50$ and $R \leq 50$. For the asymptotic regime, we need to reach large values of $R$. To this aim, we have prefered to treat the case of a discrete elliptic equation, for which the numerical simulations are exact (there is no further approximation in terms of finite element method, and the simulations are much cheaper in terms of computational cost). For the regime $R \leq 50$, we have considered a continuous equation and numerically solved the problems by a finite element method, since this is the interesting case in practice. Two cases have been considered: Periodic and quasi-periodic coefficients.

4.1. Asymptotic regime. The discrete corrector equation is

$$
-\nabla^{*} \cdot A(\xi+\nabla \phi)=0 \quad \text { in } \mathbb{Z}^{2},
$$

where for all $u: \mathbb{Z}^{2} \rightarrow \mathbb{R}$

$$
\nabla u(x):=\left[\begin{array}{c}
u\left(x+\mathbf{e}_{1}\right)-u(x) \\
u\left(x+\mathbf{e}_{2}\right)-u(x)
\end{array}\right], \nabla^{*} u(x):=\left[\begin{array}{c}
u(x)-u\left(x-\mathbf{e}_{1}\right) \\
u(x)-u\left(x-\mathbf{e}_{2}\right)
\end{array}\right],
$$

and

$$
A(x):=\operatorname{diag}\left[a\left(x, x+\mathbf{e}_{1}\right), a\left(x, x+\mathbf{e}_{2}\right)\right] .
$$

The matrix $A$ is $[0,4)^{2}$-periodic, and sketched on a periodic cell on Figure 1 . In the example considered, $a\left(x, x+\mathbf{e}_{1}\right)$ and $a\left(x, x+\mathbf{e}_{2}\right)$ represent the conductivities 1 or 100 of the horizontal edge $\left[x, x+\mathbf{e}_{1}\right]$ and the vertical edge $\left[x, x+\mathbf{e}_{2}\right]$ respectively, according to the colors on Figure 1. The homogenization theory for such discrete elliptic operators is similar to the continuous case (see for instance [21] in two dimensions, and [2] in the general 


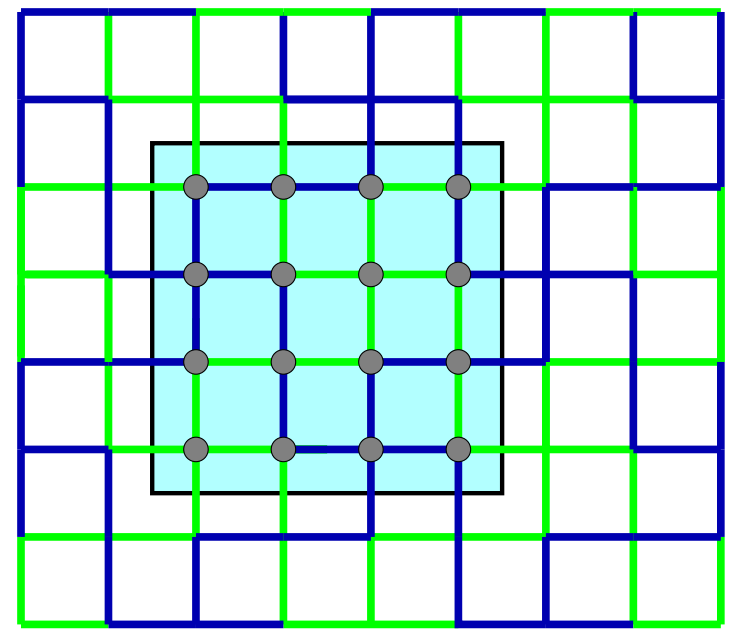

Figure 1. Periodic cell in the discrete case

TABLE 1. Order of convergence: predictions and numerical results.

\begin{tabular}{|c|c|c|c|c|c|c|c|c|c|c|}
\hline & \multicolumn{2}{|c|}{$T=\infty$} & \multicolumn{2}{c|}{$T \sim R$} & \multicolumn{2}{c|}{$T \sim R^{3 / 2}$} & \multicolumn{2}{c|}{$T \sim R^{7 / 4}$} & \multicolumn{2}{c|}{$T \sim R^{2}(\ln R)^{-8}$} \\
\hline & pred. & test & pred. & test & pred. & test & pred. & test & pred. & test \\
\hline $\mathrm{p}=0$ & 1 & 1 & 1 & 1 & 1 & 1 & 1 & 1 & 1 & 1 \\
\hline $\mathrm{p}=\infty$ & 1 & 1 & 2 & 2 & 3 & 3.1 & 3.5 & 3.4 & $4^{-}$ & $\simeq 3$ \\
\hline
\end{tabular}

case). By symmetry arguments, the homogenized matrix associated with $A$ is a multiple of the identity. It can be evaluated numerically (note that we do not make any other error than the machine precision). Its numerical value is $A_{\text {hom }}=26.240099009901 \ldots$.

In order to illustrate the discrete counterpart to Theorem 1 (which is similar, both in terms of estimates and proof, cf. [10, 11, 12] for related arguments), we have conducted the following series of tests. We have considered:

(a) Five values for the zero-order term: $T=\infty$ (no zero-order term), $T \sim R, T \sim R^{3 / 2}$, $T \sim R^{7 / 4}$, and $T \sim R^{2}(\ln R)^{-4}$

(b) Two different filters: Orders $p=0$ (no filter) and $p=\infty$;

(c) $L=R / 3$.

The predictions of Theorem 1 in terms of convergence rate of $A_{T, R, L}$ to $A_{\text {hom }}$ in function of $R$ are gathered and compared to the results of numerical tests in Table 1. More details are also given on Figures 2-6, where the overall error

$$
\operatorname{Error}(T, R):=\left|A_{\text {hom }}-A_{T, L, R}\right|
$$

is plotted in $\log$ scale in function of $R$, according to (a), (b) \& (c). Let us quickly comment on the values of $T$ in Figures 2-6. For the five dependences of $T$ upon $R$ in (a), we have chosen the prefactors so that their values roughly coincide for $R=100$. The numerical results widely confirm the analysis, and perfectly illustrate the specific influences of the two parameters $p$ and $T$. The convergence rate for $T \sim R^{2}(\ln R)^{-4}$ does not seem to meet the theoretical prediction. Indeed, the effect of the logarithm to the power 8 is still not negligeable for $R=10^{4}$ : The asymptotic regime is not yet captured by the tests. 


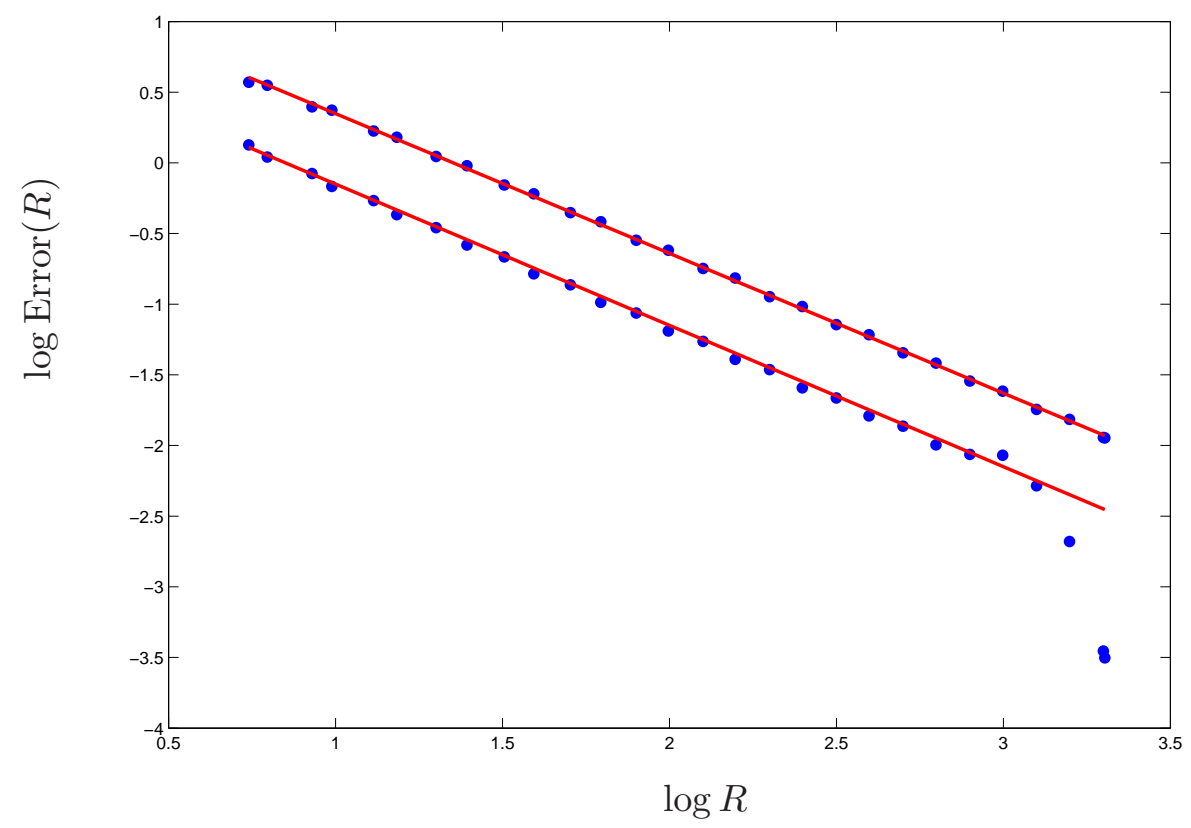

Figure 2. Absolute error in log scale without zero order term, no filter (slope -1 ), infinite order filter (slope -1 , better prefactor).

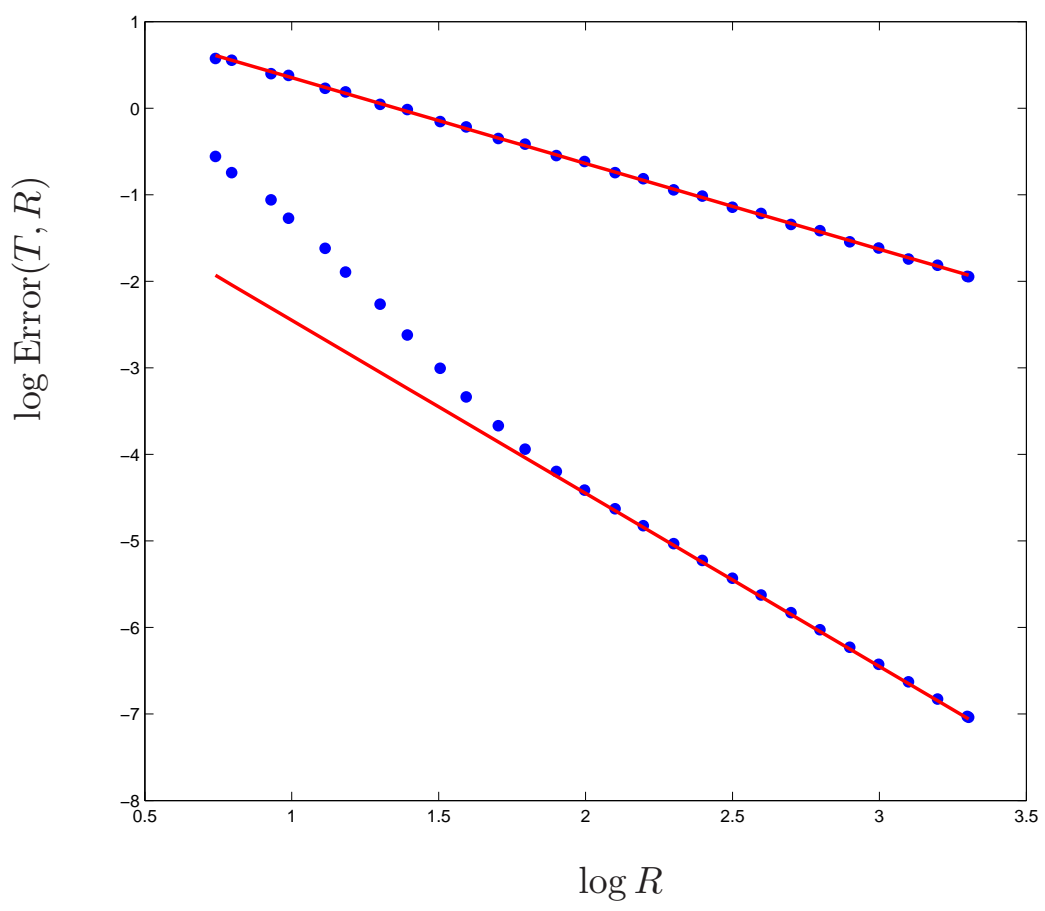

Figure 3. Absolute error in $\log$ scale for $T=R / 100$, no filter (slope -1 ), infinite order filter (slope -2 ). 


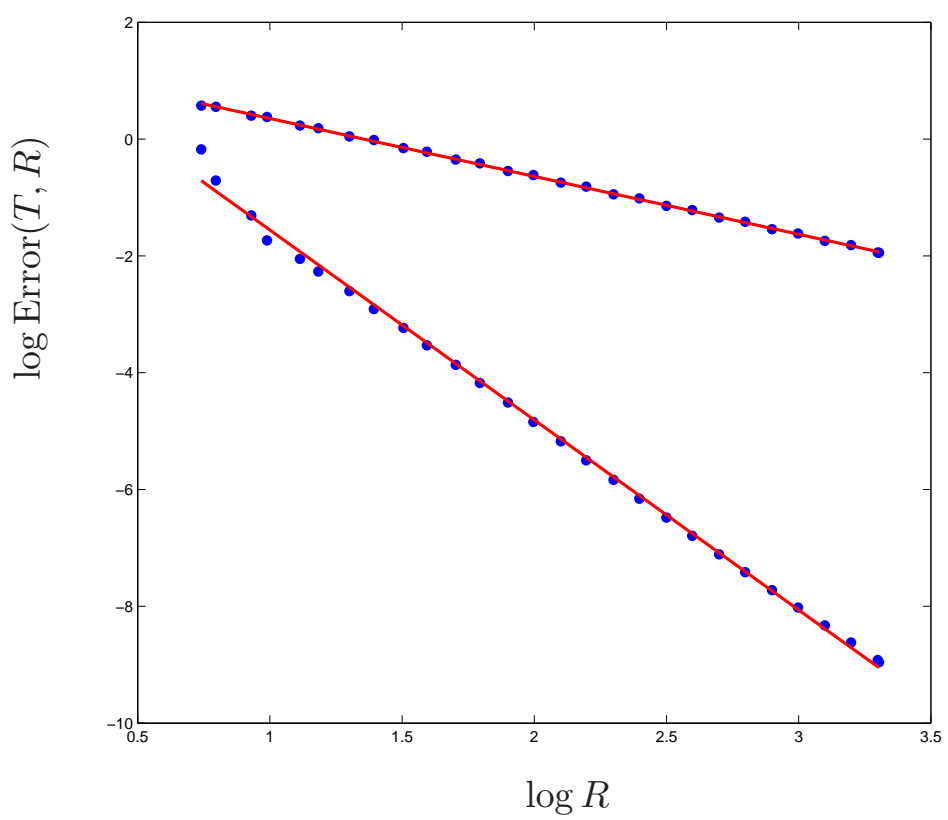

Figure 4. Absolute error in $\log$ scale for $T=R^{3 / 2} / 1000$, no filter (slope -1 ), infinite order filter (slope -3.1 ).

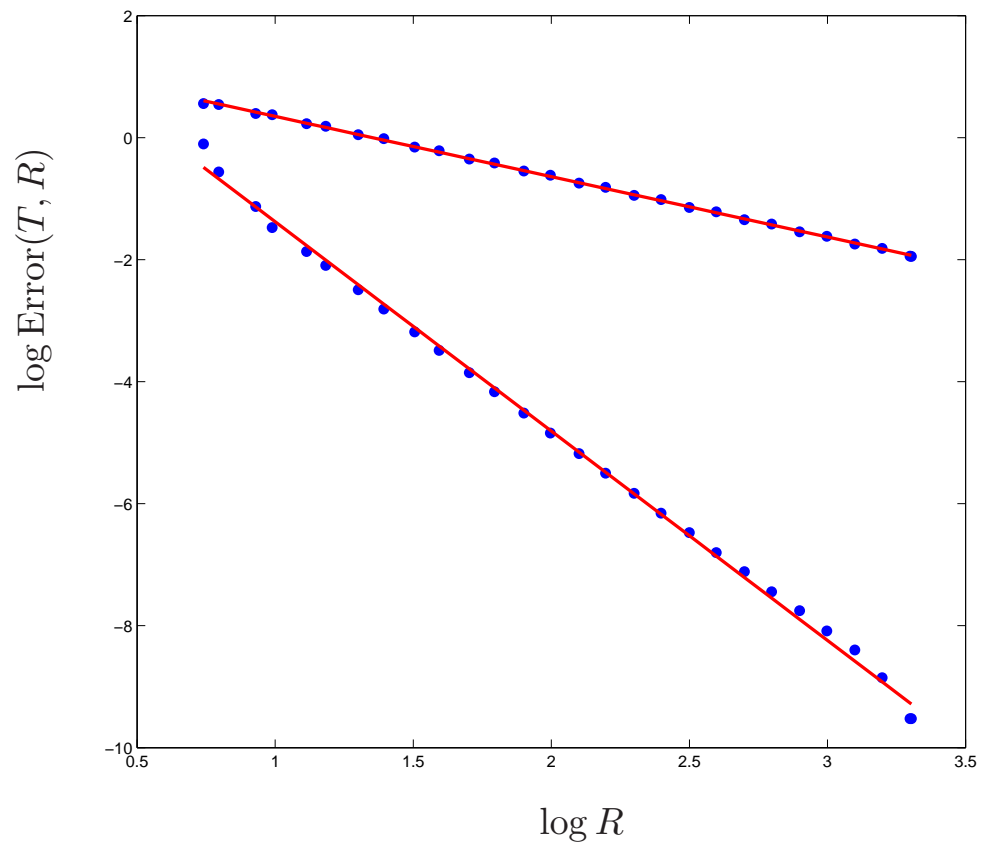

Figure 5. Absolute error in log scale for $T=R^{7 / 4} / 5000$, no filter (slope -1 ), infinite order filter (slope -3.4). 


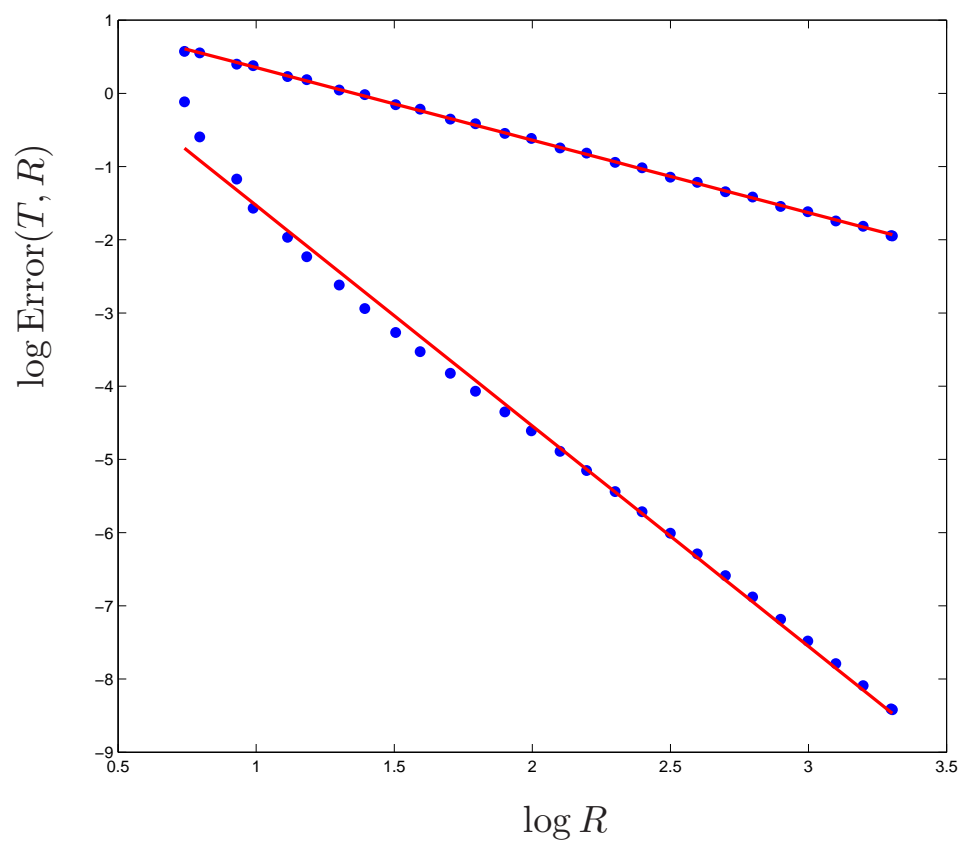

Figure 6. Absolute error in $\log$ scale for $T=R^{2} /\left(25 \ln ^{4} R\right)$, no filter (slope -1 ), infinite order filter (slope -3 ).

For similar numerical tests in the stochastic case, we refer the reader to [12], where the model and the results are described in detail. Note that, there again, the role of the zero-order term $T$ is very important in practice.

4.2. Regime $R \leq 50$. As can be seen on the previous numerical tests, the asymptotic regime is not met for small values of $R$. For instance, in the case $T=R / 100$, the apparent rate of convergence on Figure 3 is closer to 4 than to 2 up to $R \simeq 100$, whereas it is clearly 2 asymptotically. In this subsection, we focus on continuous differential operators in the regime $R \leq 50$. Since the multiplicative constants and coefficients in estimate (3.1) only depend on the dimension, and on the ellipticity and continuity constants $\alpha$ and $\beta$ of $A$, there exists a choice of the parameter $T$ in function of $R$ which is efficient for a wide class of coefficients $A$.

We consider the following matrix $A$ :

$$
A(x)=\left(\frac{2+1.8 \sin \left(2 \pi x_{1}\right)}{2+1.8 \cos \left(2 \pi x_{2}\right)}+\frac{2+\sin \left(2 \pi x_{2}\right)}{2+1.8 \cos \left(2 \pi x_{1}\right)}\right) \mathrm{Id},
$$

used as benchmark tests in [17] and [3], and for which $\alpha \simeq 0.35, \beta \simeq 20.5$, and $A_{\text {hom }} \simeq$ 2.75 Id. We take $L=R / 3, T=R / 10$ and a filter of order 2 . The global error $\mid A_{T, R, L}-$ $A_{\text {hom }}$ and the error without zero order term and without filtering are plotted on Figures 7 \& 8. Without zero-order term, the convergence rate is $R^{-1}$ as expected, and the use of a filtering method reduces the prefactor but does not change the rate. With the zeroorder term and the filtering method, the apparent convergence rate is $R^{-3}$ (note that the asymptotic theoretical rate $R^{-2}$ is not attained yet), which coincides with the convergence 


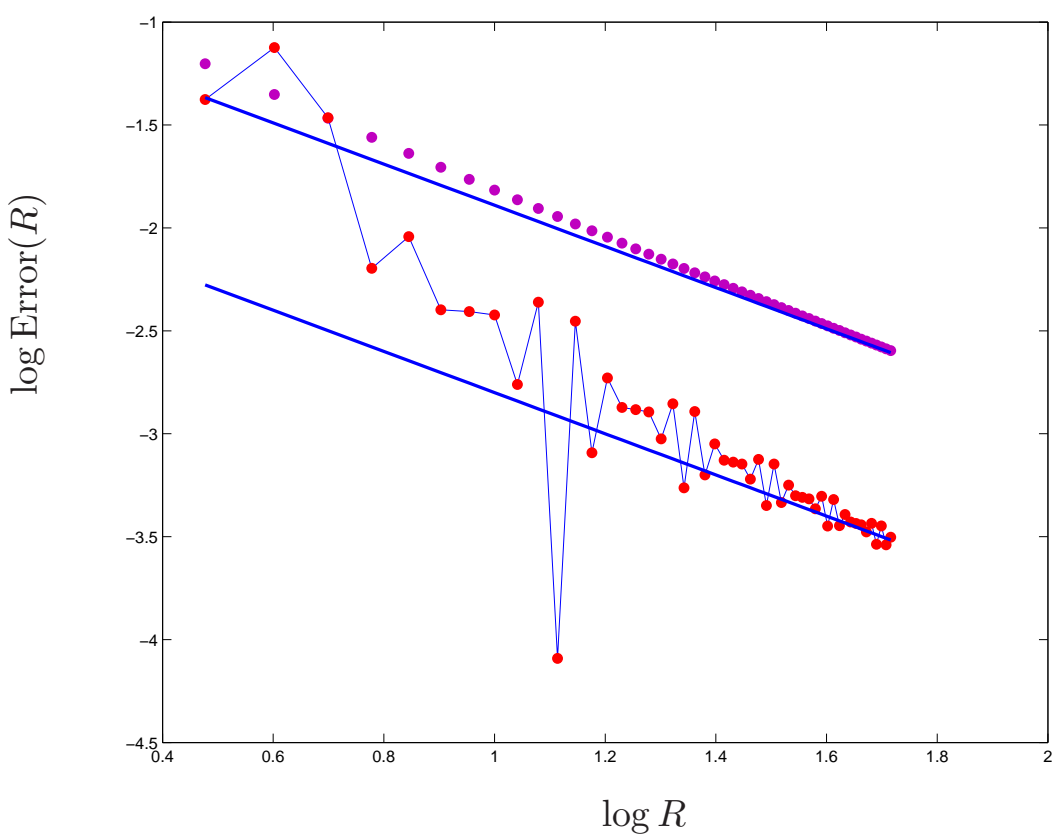

Figure 7. Error in log-log for (4.2) in function of $R \in[3,52]$ without zero-order term, with and without filtering: Slope -1 in both cases.

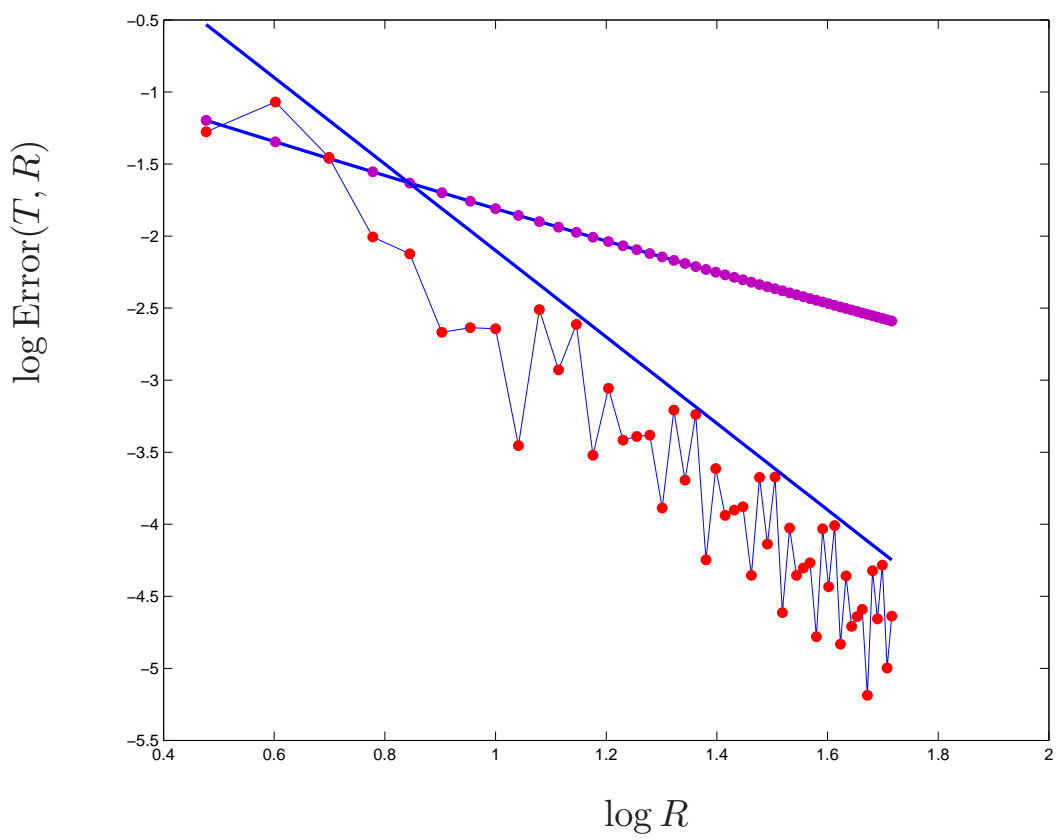

Figure 8. Error in log-log for (4.2) in function of $R \in[3,52]$ with a zeroorder term $T=R / 10$, with and without filtering: Slopes -1 and -3 . 
rate associated with filters of order 2 (cf. Lemma 1). This is in agreement with the tests in the discrete case, and confirms the analysis.

Let us now consider the following other matrix:

$$
A(x)=\left(1+30\left(2+\sin \left(2 \pi x_{1}\right) \sin \left(2 \pi x_{2}\right)\right)\right) \text { Id, }
$$

for which $\alpha=31, \beta=91$, and $A_{\text {hom }} \simeq 59.1 \mathrm{Id}$. This example is much easier to deal with than the previous one (the homogenized coefficient is close to the arithmetic mean 61). Hence, Dirichlet boundary conditions are expected to perform well, even without zero-order term. This is confirmed by the numerical tests (see Figure 9). Interestingly, the result seems to be better without filtering for $T=\infty$ in that case (such a behavior has also been reported by $\mathrm{E}$ and Yue in [22]). Adding now the zero order term with $T=R / 200, R / 300, R / 500$, the results are better provided the use of a filtering method (order 2), as can be seen on Figures 10-12. Note that the homogenized coefficient is 22 times larger than in the previous case, so that we could expect $T$ to be 22 times smaller than in the case (4.2).

These two series of tests clearly show that the numerical method performs quite well in this continuous periodic setting, even with a limited number of periodic cells.

Besides the periodic and stochastic settings, another standard benchmark case is the (academic) quasi-periodic setting. The last series of tests is dedicated to this case, and we consider the following quasi-periodic coefficients used in [3]:

$$
A(x)=\left(\begin{array}{cc}
4+\cos \left(2 \pi\left(x_{1}+x_{2}\right)\right)+\cos \left(2 \pi \sqrt{2}\left(x_{1}+x_{2}\right)\right) & 0 \\
0 & 6+\sin ^{2}\left(2 \pi x_{1}\right)+\sin ^{2}\left(2 \pi \sqrt{2} x_{1}\right)
\end{array}\right) .
$$

In this case, the homogenized coefficients are not easy to compute. They can only be extrapolated. We have taken for the approximation of the homogenized coefficients (that we call coefficient of reference) the output of the computation with $T=R / 100$ and $R=52$. Although this may introduce a bias in favour of the proposed strategy, it can be checked a posteriori: The method without zero-order term and without filtering is expected to converge at a rate $R^{-1}$. This is effectively what we observe on Figure 13 using this coefficient of reference. Instead, if we use as a reference the output of the computation for $R=52$ without zero-order term nor filtering, then we observe a super-linear convergence which is artificial (see Figure 13). With the proposed method, as can be seen on Figure 14, the rate of convergence seems to be much better (the slope of the straight line is -5 ). Hence the method performs also quite well for this quasi-periodic example.

To conclude, the numerical tests performed clearly demonstrate that the proposed strategy effectively reduces the resonance error, in the periodic, quasi-periodic and stochastic cases, both asymptotically and in the small number of periods regime. In addition, the analysis is sharp and both the roles of the zero-order term and of the filter are crucial.

\section{ACKNOWLEDGMENTS}

The author gratefully acknowledges the help of Michel Hua for the numerical tests. 


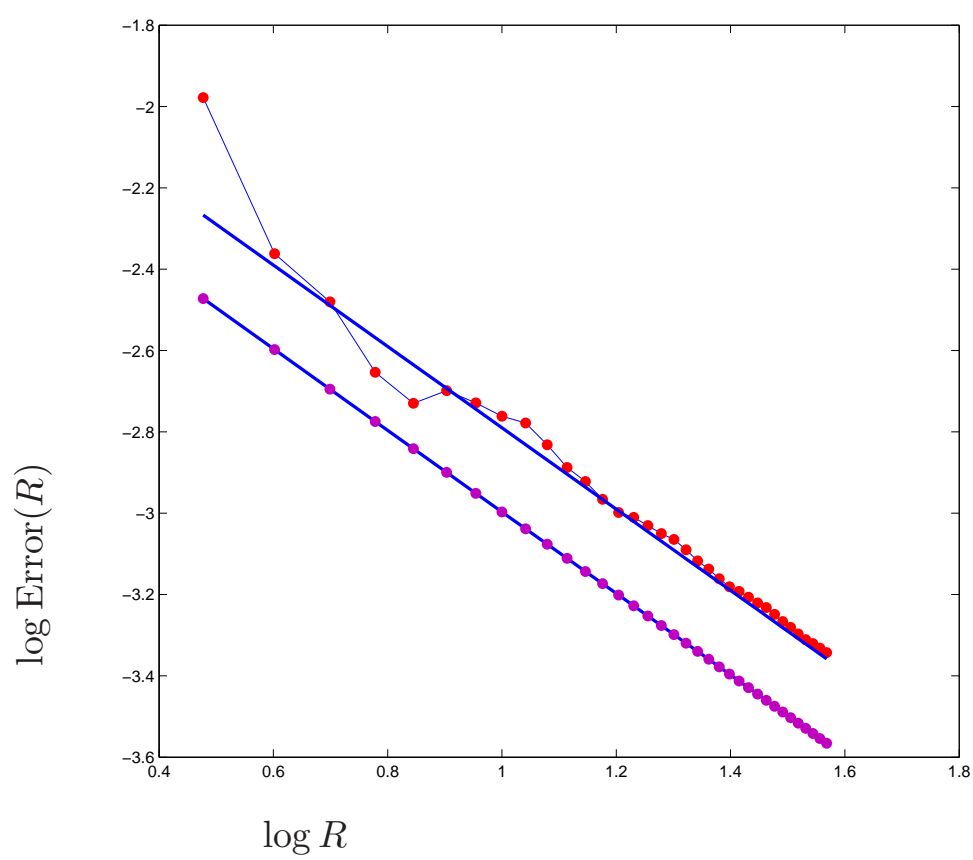

Figure 9. Error in log-log for (4.3) in function of $R \in[3,35]$ without zero-order term, with and without filtering: Slope -1 in both cases.

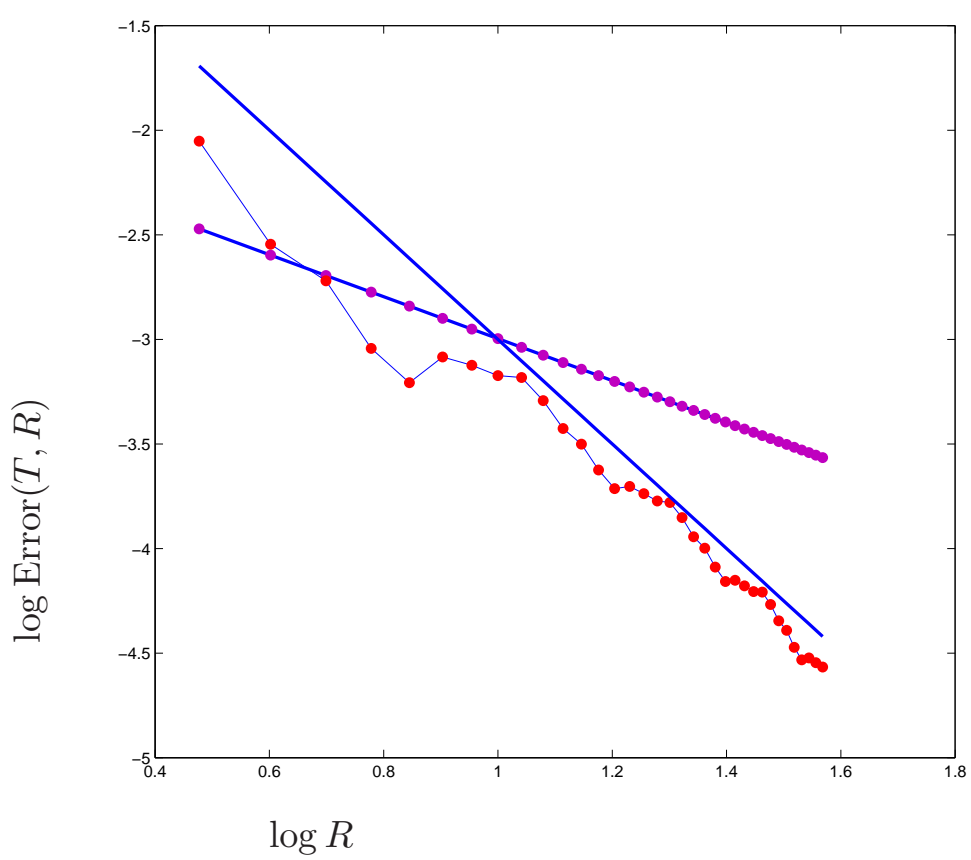

FiguRE 10. Error in $\log$-log for (4.3) in function of $R \in[3,38]$ with a zero-order term $T=R / 200$, with and without filtering: Slopes -1 and -2.5 . 


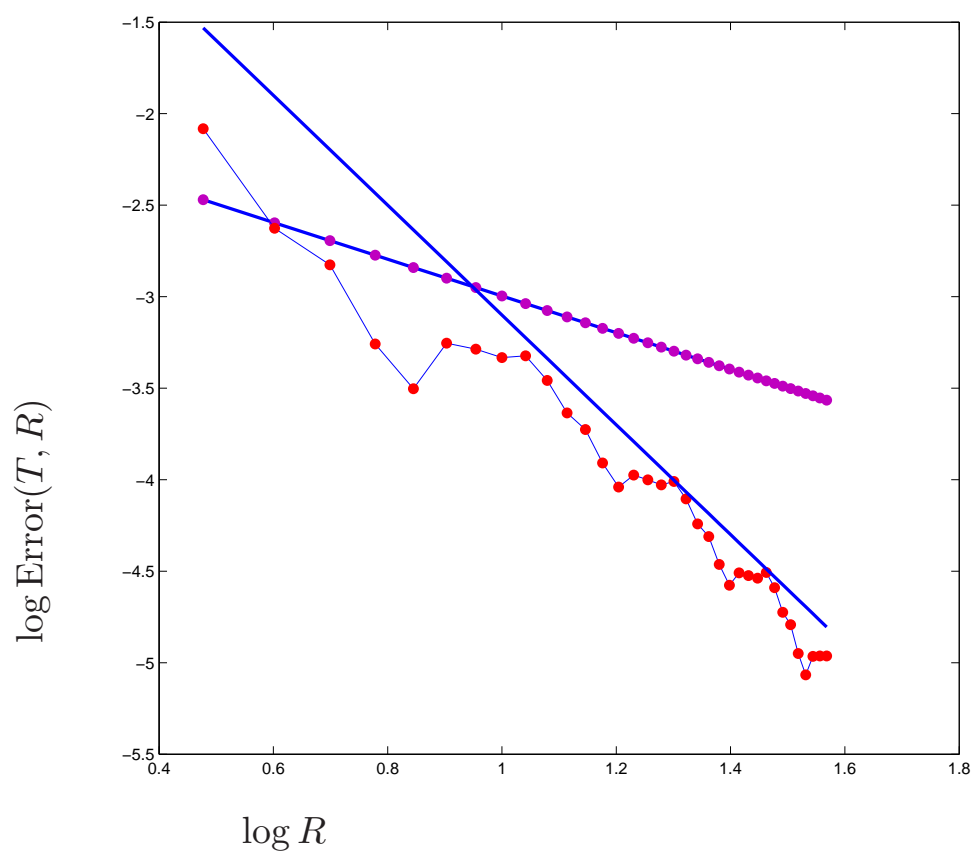

FiguRE 11. Error in log-log for (4.3) in function of $R \in[3,38]$ with a zero-order term $T=R / 300$, with and without filtering: Slopes -1 and -3 .

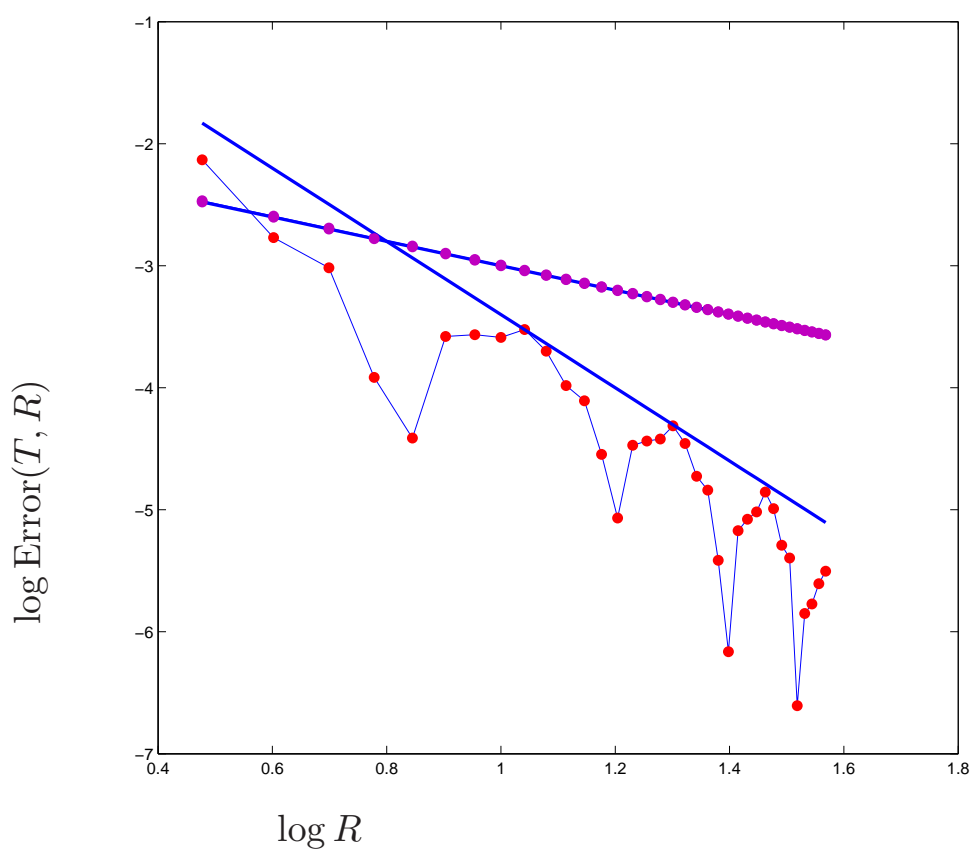

Figure 12. Error in log-log for (4.3) in function of $R \in[3,38]$ with a zero-order term $T=R / 500$, with and without filtering: Slopes -1 and -3 . 


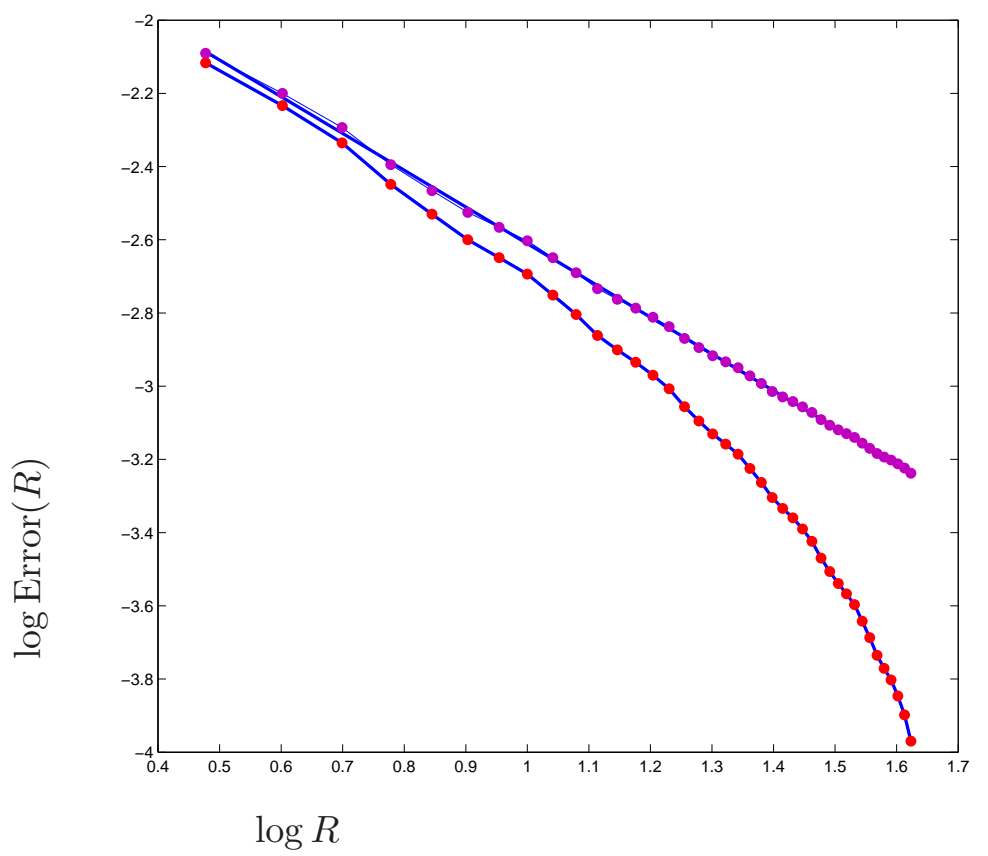

Figure 13. Error in log-log for (4.4) in function of $R \in[3,42]$ without zero-order term and without filtering, for the two different coefficients of reference: Slope -1 and artificial super-linear convergence.

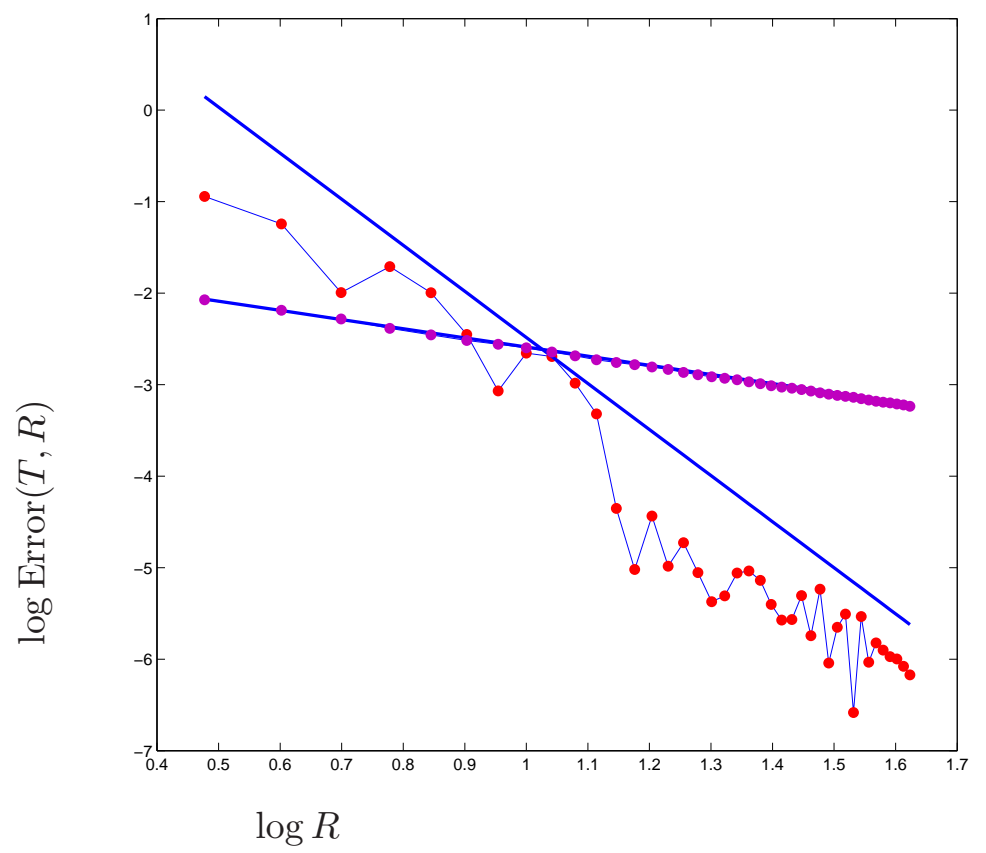

FiguRE 14. Error in $\log$-log for (4.4) in function of $R \in[3,42]$ with a zero-order term $T=R / 200$, with and without filtering: Slopes -1 and -5 . 


\section{REFERENCES}

[1] S. Agmon. Lectures on exponential decay of solutions of second-order elliptic equations: bounds on eigenfunctions of $N$-body Schrödinger operators, volume 29 of Mathematical Notes. Princeton University Press, Princeton, NJ, 1982.

[2] R. Alicandro and M. Cicalese. A general integral representation result for the continuum limits of discrete energies with superlinear growth. SIAM J. Math. Anal., 36(1):1-37, 2004.

[3] X. Blanc and C. Le Bris. Improving on computation of homogenized coefficients in the periodic and quasi-periodic settings. preprint.

[4] A. Bourgeat and A. Piatnitski. Approximations of effective coefficients in stochastic homogenization. Ann. I. H. Poincaré, 40:153-165, 2005.

[5] W. E, B. Engquist, X. Li, W. Ren, and E. Vanden-Eijnden. Heterogeneous multiscale methods: A review. Commun. Comput. Phys., 2:367-450, 2007.

[6] W. E, P.B. Ming, and P.W. Zhang. Analysis of the heterogeneous multiscale method for elliptic homogenization problems. J. Amer. Math. Soc., 18:121-156, 2005.

[7] R. E. Edwards. Fourier series. Vol. 2, volume 85 of Graduate Texts in Mathematics. Springer-Verlag, New York, second edition, 1982. A modern introduction.

[8] A. Gloria. An analytical framework for the numerical homogenization of monotone elliptic operators and quasiconvex energies. Multiscale Model. Simul., 5(3):996-1043, 2006.

[9] A. Gloria. An analytical framework for numerical homogenization - Part II: windowing and oversampling. Multiscale Model. Simul., 7(1):275-293, 2008.

[10] A. Gloria and F. Otto. An optimal variance estimate in stochastic homogenization of discrete elliptic equations. 2009. Preprint. Available at http://hal.archives-ouvertes.fr/hal-00383953/en/.

[11] A. Gloria and F. Otto. An optimal error estimate in stochastic homogenization of discrete elliptic equations. 2010. Preprint. Available at http://hal.archives-ouvertes.fr/inria-00457020/en/.

[12] A. Gloria and F. Otto. Numerical approximation of effective coefficients in stochastic homogenization of discrete elliptic equations. In preparation.

[13] M. Grüter and K.-O. Widman. The Green function for uniformly elliptic equations. Manuscripta Math., 37:303-342, 1982.

[14] Q. Han and F. Lin. Elliptic partial differential equations. Courant Institute of Mathematical Sciences, New York, 1997.

[15] T.Y. Hou and X.H Wu. A multiscale finite element method for elliptic problems in composite materials and porous media. J. Comput. Phys., 134:169-189, 1997.

[16] T.Y. Hou, X.H. Wu, and Z.Q. Cai. Convergence of a multiscale finite element method for elliptic problems with rapidly oscillating coefficients. Math. Comput., 68:913-943, 1999.

[17] T.Y. Hou, X.H. Wu, and Y. Zhang. Removing the cell resonance error in the multiscale finite element method via a petrov-galerkin formulation. Commun. Math. Sci., 2:185-205, 2004.

[18] V.V. Jikov, S.M. Kozlov, and O.A. Oleinik. Homogenization of Differential Operators and Integral Functionals. Springer-Verlag, Berlin, 1994.

[19] N. Meyers. An $L^{p}$-estimate for the gradient of solutions of second order elliptic divergence equations. Ann. Scuola Norm. Sup. Pisa Cl. Sci. (3), 17(3):189-206, 1963.

[20] F. Murat and L. Tartar. H-convergence. In A.V. Cherkaev and R.V. Kohn, editors, Topics in the Mathematical Modelling of Composites Materials, volume 31 of Progress in nonlinear differential equations and their applications, pages 21-44. Birkhäuser, 1997.

[21] M. Vogelius. A homogenization result for planar, polygonal networks. RAIRO Modél. Math. Anal. Numér., 25(4):483-514, 1991.

[22] X. Yue and W. E. The local microscale problem in the multiscale modeling of strongly heterogeneous media: effects of boundary conditions and cell size. J. Comput. Phys., 222(2):556-572, 2007.

\section{Appendix A. Proof of Lemma 1}

We first prove Lemma 1 for $d=1$, and expand $\phi$ in Fourier series:

$$
\phi(x)=\sum_{k \in \mathbb{Z}} c_{k} \exp (i 2 \pi k x) .
$$


Step 1. Proof of

$$
\left|\int_{-L}^{L} \exp (i 2 \pi k x) \mu_{L}(x) d x\right| \lesssim|k|^{-p} L^{-(p+1)},
$$

for $k \neq 0$ and $p>0$. After $p$ integrations by parts, and using Definition 1 (iii), we have

$$
\int_{-L}^{L} \exp (i 2 \pi k x) \mu_{L}(x) d x=(-1)^{p} \int_{-L}^{L} \frac{1}{(2 i \pi k)^{p}} \exp (i 2 \pi k x) L^{-p} L^{-1} \mu^{(p)}\left(L^{-1} x\right) d x .
$$

Extending $\mu$ by zero on $\mathbb{R} \backslash(-1,1)$, we may introduce

$$
\begin{aligned}
\bar{\mu}_{L}:(-1,1) & \rightarrow \mathbb{R} \\
x & \mapsto L^{-1} \sum_{k=-[L / 2]-1}^{[L / 2]+1} \mu^{(p)}\left(L^{-1}(x+2 k)\right),
\end{aligned}
$$

where $[\cdot]$ denotes the integer part. Since $\mu^{(p)}$ is Lipschitz (with a constant $C_{\mu}$ ), for all $x \in(-1,1)$ one has

$$
\left|\bar{\mu}_{L}(x)-m_{\mu}\right| \leq C_{\mu} L^{-1}|x|,
$$

where $m_{\mu}=\int_{-1}^{1} \bar{\mu}_{L}(x) d x$. Using $\bar{\mu}_{L}$ and the periodicity of $x \mapsto \exp (i 2 \pi k x)$, we rewrite (A.3) as

$$
\begin{aligned}
\left|\int_{-L}^{L} \exp (i 2 \pi k x) \mu_{L}(x) d x\right| & \stackrel{(\mathrm{A} .4)}{=}\left|\frac{L^{-p}}{(-2 i \pi k)^{p}} \int_{-1}^{1} \exp (i 2 \pi k x) \bar{\mu}_{L}(x) d x\right| \\
& \stackrel{(\text { A.5) }}{\leq} \frac{C_{\mu} L^{-(p+1)}}{(2 \pi k)^{p}}
\end{aligned}
$$

which is (A.2).

Step 2. Proof of (3.12) for $d=1$. For $p=0,(3.12)$ is trivial, and we only consider $p>0$. We first note that (A.1) and Definition 1 (ii) imply

$$
\int_{-L}^{L} \phi(x) \mu_{L}(x) d x-\mathcal{M}(\phi)=\sum_{k \in \mathbb{Z} \backslash\{0\}} c_{k} \int_{-L}^{L} \exp (i 2 \pi k x) \mu_{L}(x) d x .
$$

Combined with (A.2), this turns into

$$
\left|\int_{-L}^{L} \phi(x) \mu_{L}(x) d x-\mathcal{M}(\phi)\right| \lesssim L^{-(p+1)} \sum_{k \in \mathbb{Z} \backslash\{0\}} c_{k} k^{-p} .
$$

Using then Cauchy-Schwarz' inequality and Parseval's identity, one concludes

$$
\begin{aligned}
\left|\int_{-L}^{L} \phi(x) \mu_{L}(x) d x-\mathcal{M}(\phi)\right| & \lesssim L^{-(p+1)}\left(\sum_{k \in \mathbb{Z} \backslash\{0\}} c_{k}^{2}\right)^{1 / 2}\left(\sum_{k \in \mathbb{Z} \backslash\{0\}} k^{-2 p}\right)^{1 / 2} \\
& \lesssim L^{-(p+1)}\|\phi\|_{L^{2}(0,1)}
\end{aligned}
$$

since $p \geq 1$.

Step 3. Extension to dimension $d>1$. We use a Fourier expansion as above:

$$
\phi(x)=\sum_{k_{1}, \ldots, k_{d} \in \mathbb{Z}} c_{k_{1}, \ldots, k_{d}} \exp (i 2 \pi k \cdot x),
$$


where $k=\left(k_{1}, \ldots, k_{d}\right)$ and $x=\left(x_{1}, \ldots, x_{d}\right)$. In this case, (A.2) is replaced by

$$
\left|\int_{Q_{L}} \exp (i 2 \pi k \cdot x) \mu_{L}(x) d x\right| \lesssim L^{-(p+1)} \prod_{l=1}^{d}\left(1+\left|k_{l}\right|\right)^{-p}
$$

for all $k \neq 0$. As in Step 3, this leads to

$$
\begin{aligned}
\left|\int_{Q_{L}} \phi(x) \mu_{L}(x) d x-\mathcal{M}(\phi)\right| & \lesssim L^{-(p+1)}\left(\sum_{k \in \mathbb{Z}^{d}} c_{k}^{2}\right)\left(\sum_{k \in \mathbb{Z}^{d}} \prod_{l=1}^{d}\left(1+\left|k_{l}\right|\right)^{-2 p}\right) \\
& \lesssim L^{-(p+1)}\|\phi\|_{L^{2}(Q)} .
\end{aligned}
$$

Step 4. The case $\phi \in L^{q}(Q), 1<q<2$. In this case, one cannot use Parseval's identity anylonger. For $p \geq 2$, it is enough to use $\left|c_{k}\right| \leq\|\phi\|_{L^{1}(Q)}$ and the summability of $\prod_{l=1}^{d}(1+$ $\left.\left|k_{l}\right|\right)^{-2}$ on $\mathbb{Z}^{d}$ to conclude. For $p=1$, we appeal to Hardy-Littlewood's inequality (see for instance $[7,13.11 .1])$ for functions in $L^{q}(Q), 1<q<2$ :

$$
\left(\sum_{k \in \mathbb{Z}^{d}} \prod_{l=1}^{d}\left(1+\left|k_{l}\right|\right)^{q-2} c_{k}^{q}\right)^{1 / q} \lesssim\|\phi\|_{L^{q}(Q)},
$$

and conclude by Hölder's inequality with exponents $(q /(q-1), q)$

$$
\begin{aligned}
& \left|\int_{-L}^{L} \phi(x) \mu_{L}(x) d x-\mathcal{M}(\phi)\right| \\
& \quad \lesssim L^{-(p+1)} \sum_{k \in \mathbb{Z}^{d}} \prod_{l=1}^{d}\left(1+\left|k_{l}\right|\right)^{-1} c_{k} \\
& \quad=L^{-(p+1)} \sum_{k \in \mathbb{Z}^{d}} \prod_{l=1}^{d}\left(1+\left|k_{l}\right|\right)^{-2(q-1) / q}\left(1+\left|k_{l}\right|\right)^{(q-2) / q} c_{k} \\
& \quad \leq L^{-(p+1)}\left(\sum_{k \in \mathbb{Z}^{d}} \prod_{l=1}^{d}\left(1+\left|k_{l}\right|\right)^{-2}\right)^{(q-1) / q}\left(\sum_{k \in \mathbb{Z}^{d}} \prod_{l=1}^{d}\left(1+\left|k_{l}\right|\right)^{q-2} c_{k}^{q}\right)^{1 / q} \\
& \quad \lesssim L^{-(p+1)}\|\phi\|_{L^{q}(Q)} .
\end{aligned}
$$

\section{Appendix B. Proof of Lemma 2}

The following proof is standard and relies on three arguments:

- Harnack's inequality,

- Pointwise estimates for the Green function of second order elliptic equations,

- The operator positivity method due to Agmon (see [1]).

W. 1. o. g. we assume $y=0$, and use the shorthand notation $G_{T}(x)$ for $G_{T}(x, y)$. 


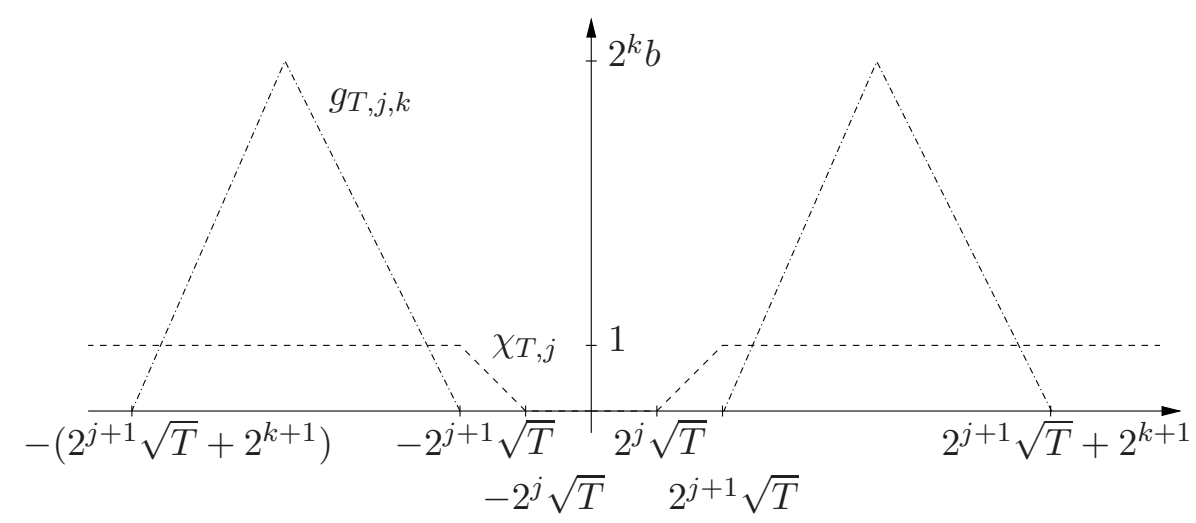

FIgURE 15. Functions $g_{T, j, k}$ and $\chi_{T, j}$ for $d=1$.

Step 1. Operator positivity method. Let $b>0$, and for all $j, k \in \mathbb{N}$ let $\chi_{T, j}: \mathbb{R}^{d} \rightarrow \mathbb{R}^{+}$ and $g_{T, j, k}: \mathbb{R}^{d} \rightarrow \mathbb{R}^{+}$be given by

$$
\chi_{T, j}(x)= \begin{cases}\text { for }|x| \leq 2^{j} \sqrt{T} & : \\ \text { for } 2^{j} \sqrt{T} \leq|x| \leq 2^{j+1} \sqrt{T} & :\left(2^{j} \sqrt{T}\right)^{-1}\left(|x|-2^{j} \sqrt{T}\right) \\ \text { for }|x| \geq 2^{j+1} \sqrt{T} & :\end{cases}
$$

and

$$
g_{T, j, k}(x)=\left\{\begin{array}{ll}
\text { for }|x| \leq 2^{j+1} \sqrt{T} & : 0 \\
\text { for } 2^{j+1} \sqrt{T} \leq|x| \leq 2^{j+1} \sqrt{T}+2^{k} & : b\left(|x|-2^{j+1} \sqrt{T}\right) \\
\text { for } 2^{j+1} \sqrt{T}+2^{k} \leq|x| \leq 2^{j+1} \sqrt{T}+2^{k+1} & : 2^{k+1} b+b\left(2^{j+1} \sqrt{T}-|x|\right) \\
\text { for } 2^{j+1} \sqrt{T}+2^{k+1} \leq|x| & : 0
\end{array} .\right.
$$

These functions are plotted for convenience on Figure 15 for $d=1$.

We multiply the defining equation for $G_{T}$ by the test function

$$
x \mapsto \chi_{T, j}(x)^{2} \exp \left(2 g_{T, j, k}(x)\right) G_{T}(x)
$$

and integrate on $\mathbb{R}^{d}$, obtaining

$$
\begin{aligned}
& T^{-1} \int_{\mathbb{R}^{d}}\left(\chi_{T, j}(x) \exp \left(g_{T, j, k}(x)\right) G_{T}(x)\right)^{2} d x \\
& \quad+\int_{\mathbb{R}^{d}} \nabla\left(\chi_{T, j}(x)^{2} \exp \left(2 g_{T, j, k}(x)\right) G_{T}(x)\right) \cdot A(x) \nabla G_{T}(x) d x=0 .
\end{aligned}
$$

We focus on the second term of the equation and use Leibniz' rule. For the sake of clarity, we drop the subscripts and variables in the following calculation.

$$
\begin{aligned}
\nabla & \left(\chi^{2} \exp (2 g) G\right) \cdot A \nabla G \\
= & \nabla(\chi \exp (g) G) \cdot A \chi \exp (g) \nabla G+\chi \exp (g) G \nabla(\chi \exp (g)) \cdot A \nabla G \\
= & \nabla(\chi \exp (g) G) \cdot A \nabla(\chi \exp (g) G) \\
& \underbrace{-\nabla(\chi \exp (g) G) \cdot A \nabla(\chi \exp (g)) G}_{\text {? }}+\chi \exp (g) G \nabla(\chi \exp (g)) \cdot A \nabla G .
\end{aligned}
$$


We rewrite the second term of the $\mathrm{r}$. h. s. as follows:

$$
\begin{aligned}
\boldsymbol{\alpha} & =-\chi \exp (g) \nabla G \cdot A \nabla(\chi \exp (g)) G-G^{2} \nabla(\chi \exp (g)) \cdot A \nabla(\chi \exp (g)) \\
& =-G^{2} \nabla(\chi \exp (g)) \cdot A \nabla(\chi \exp (g))-\chi \exp (g) G \nabla(\chi \exp (g)) \cdot A \nabla G
\end{aligned}
$$

by symmetry of $A$. The combination of these two identities yields

$$
\begin{aligned}
& \nabla\left(\chi^{2} \exp (2 g) G\right) \cdot A \nabla G \\
& \quad=\nabla(\chi \exp (g) G) \cdot A \nabla(\chi \exp (g) G)-G^{2} \nabla(\chi \exp (g)) \cdot A \nabla(\chi \exp (g)) \\
& \quad \geq-\beta G^{2}|\nabla(\chi \exp (g))|^{2}
\end{aligned}
$$

by the uniform bound on $A$. Setting $\psi_{T, j, k}: x \mapsto \exp \left(g_{T, j, k}(x)\right) G_{T}(x)$, we insert the latter inequality into (B.1) to get

$$
\int_{\mathbb{R}^{d}}\left(T^{-1} \chi_{T, j}(x)^{2} \psi_{T, j, k}(x)^{2}-\beta G_{T}(x)^{2}\left|\nabla\left(\chi_{T, j}(x) \exp \left(g_{T, j, k}(x)\right)\right)\right|^{2}\right) d x \leq 0 .
$$

Using the properties of $\chi_{T, j}$ and $g_{T, j, k}$, this turns into

$$
\begin{aligned}
& \int_{|x| \geq 2^{j+1} \sqrt{T}}\left(T^{-1} \psi_{T, j, k}(x)^{2}-\beta G_{T}(x)^{2}\left|\nabla \exp \left(g_{T, j, k}(x)\right)\right|^{2}\right) d x \\
& \quad \leq \int_{2^{j} \sqrt{T} \leq|x|<2^{j+1} \sqrt{T}} \beta\left|\nabla \chi_{T, j}(x)\right|^{2} G_{T}(x)^{2} d x,
\end{aligned}
$$

and finally

$\int_{|x| \geq 2^{j+1} \sqrt{T}}\left(T^{-1}-\beta\left|\nabla g_{T, j, k}(x)\right|^{2}\right) \psi_{T, j, k}(x)^{2} d x \leq \beta T^{-1} 2^{-2 j} \int_{2^{j} \sqrt{T} \leq|x|<2^{j+1} \sqrt{T}} G_{T}(x)^{2} d x$.

Choosing $b=(2 \beta T)^{-1 / 2}$ then yields

$$
\int_{|x| \geq 2^{j+1} \sqrt{T}} \psi_{T, j, k}(x)^{2} d x \lesssim 2^{-2 j} \int_{2^{j} \sqrt{T} \leq|x|<2^{j+1} \sqrt{T}} G_{T}(x)^{2} d x .
$$

We then pass to the limit $k \rightarrow \infty$ by the monotone convergence theorem to obtain

$$
\int_{|x| \geq 2^{j+1} \sqrt{T}} \exp \left(2 b\left(|x|-2^{j+1} \sqrt{T}\right)\right) G_{T}(x)^{2} d x \lesssim 2^{-2 j} \int_{2^{j} \sqrt{T} \leq|x|<2^{j+1} \sqrt{T}} G_{T}(x)^{2} d x,
$$

and therefore

$$
\int_{|x| \geq 2^{j+2} \sqrt{T}} G_{T}(x)^{2} d x \lesssim 2^{-2 j} \exp \left(-b 2^{j+2} \sqrt{T}\right) \int_{2^{j} \sqrt{T} \leq|x|<2^{j+1} \sqrt{T}} G_{T}(x)^{2} d x .
$$

Step 2. Decay estimates. We now appeal to standard decay estimates derived via the De Giorgi-Nash-Moser theory. We refer to [13, Theorem 1.1] for $d>2$. For $d=2$, we refer to $[10$, Lemma 8 (i)\&(iii)], whose proof is actually first presented in the continuous case (and later on adapted to the discrete case). In particular, for all $j \in \mathbb{N}$ and $d \geq 2$, we have

$$
\int_{2^{j} \sqrt{T} \leq|x|<2^{j+1} \sqrt{T}} G_{T}(x)^{2} d x \lesssim\left(2^{j} \sqrt{T}\right)^{d}\left(\left(2^{j} \sqrt{T}\right)^{2-d}\right)^{2}=\left(2^{j} \sqrt{T}\right)^{4-d} .
$$

Note that the pointwise estimates in [13, Theorem 1.1] for $G_{T}$ are uniform in $T$ for $d>2$, so that (B.3) indeed holds on any annulus of the form $\left\{2^{j} R \leq|x|<2^{j+1} R\right\}, R \geq 1$. 
This is not the case for $d=2$ (the Green function of the Laplace operator diverges logarithmically).

Step 3. Harnack's inequality. We rewrite the defining equation for $G_{T}$ as

$$
-\nabla \cdot A \nabla G_{T}(x)=-T^{-1} G_{T}(x) \leq 0, \quad \text { for }|x| \geq 1,
$$

so that we may use Harnack's inequality for non-negative subsolutions (see for instance [14, Theorem 4.14]):

$$
\sup _{2^{j} \sqrt{T} \leq|x| \leq 2^{j+1} \sqrt{T}} G_{T}(x) \lesssim\left(2^{j} \sqrt{T}\right)^{-d / 2}\left\|G_{T}\right\|_{L^{2}\left(\left\{2^{j-1} \sqrt{T} \leq|x| \leq 2^{j+2} \sqrt{T}\right\}\right)} .
$$

The combination of (B.2), (B.3) \& (B.4) concludes the proof of the lemma.

(Antoine Gloria) Projet SIMPAF, INRIA Lille-Nord Europe, France

E-mail address: antoine.gloria@inria.fr 